\title{
Intelligent Fruit Yield Estimation for Orchards Using Deep Learning Based Semantic Segmentation Techniques-A Review
}

\section{OPEN ACCESS}

Edited by:

Salvatore Filippo Di Gennaro, Institute for Bioeconomy, National

Research Council (CNR), Italy

Reviewed by:

Giovanni Avola,

Institute for Bioeconomy, National

Research Council (CNR), Italy

Michael Gomez Selvaraj,

Consultative Group on International

Agricultural Research (CGIAR),

United States

*Correspondence:

Purushothaman Raja

raja_sastra@yahoo.com

Specialty section:

This article was submitted to Technical Advances in Plant Science, a section of the journal

Frontiers in Plant Science

Received: 23 March 2021

Accepted: 31 May 2021

Published: 25 June 2021

Citation:

Maheswari P, Raja P, Apolo-Apolo OE and Pérez-Ruiz M (2021) Intelligent Fruit Yield Estimation

for Orchards Using Deep Learning Based Semantic Segmentation

Techniques-A Review.

Front. Plant Sci. 12:684328. doi: 10.3389/fp/s.2021.684328

\author{
Prabhakar Maheswari1, Purushothaman Raja ${ }^{1 *}$, Orly Enrique Apolo-Apolo² and \\ Manuel Pérez-Ruiz² \\ ${ }^{1}$ School of Mechanical Engineering, SASTRA Deemed University, Thanjavur, India, ${ }^{2}$ Departamento de Ingeniería \\ Aeroespacial y Mecánica de Fluidos, Área de Ingeniería Agroforestal, Universidad de Sevilla, Seville, Spain
}

Smart farming employs intelligent systems for every domain of agriculture to obtain sustainable economic growth with the available resources using advanced technologies. Deep Learning (DL) is a sophisticated artificial neural network architecture that provides state-of-the-art results in smart farming applications. One of the main tasks in this domain is yield estimation. Manual yield estimation undergoes many hurdles such as labor-intensive, time-consuming, imprecise results, etc. These issues motivate the development of an intelligent fruit yield estimation system that offers more benefits to the farmers in deciding harvesting, marketing, etc. Semantic segmentation combined with DL adds promising results in fruit detection and localization by performing pixel-based prediction. This paper reviews the different literature employing various techniques for fruit yield estimation using DL-based semantic segmentation architectures. It also discusses the challenging issues that occur during intelligent fruit yield estimation such as sampling, collection, annotation and data augmentation, fruit detection, and counting. Results show that the fruit yield estimation employing DL-based semantic segmentation techniques yields better performance than earlier techniques because of human cognition incorporated into the architecture. Future directions like customization of $\mathrm{DL}$ architecture for smart-phone applications to predict the yield, development of more comprehensive model encompassing challenging situations like occlusion, overlapping and illumination variation, etc., were also discussed.

Keywords: precision agriculture, yield estimation, deep learning, semantic segmentation, fruit detection and localization

\footnotetext{
Abbreviations: AI, Artificial Intelligence; AVIRIS, Airborne Visible/Infrared Imaging Spectrometer; CCD, Charge Coupled Device; CNN, Convolutional Neural Networks; CPU, Central Processing Unit; CART, Classification and Regression Trees Classifier; CMOS, Complementary Metal-Oxide-Semiconductor; CRAID, CRanberry Aerial Image Dataset; CRF, Conditional Random Fields; DL, Deep learning; ECa, apparent Electrical Conductivity; EM, Expectation-Maximization; FCN, Fully Convolutional Networks; GFPN, Gate Feature Pyramid Network; GMM, Gaussian Mixture Model; GPU, Graphics Processing Unit; HOG, Histograms of Oriented Gradient; IoU, Intersection over Union; LSTM, Long Short-Term Memory; MIL, Multiple Instance Learning; ML, Machine Learning; MRF, Markov Random Fields; NDVI, Normalized Difference Vegetation Index; NMS, Non-Maximum Suppression; PA, Precision Agriculture; R-CNN, Regions with Convolutional Neural Networks; ReLU, Rectified Linear Unit; ResNet, Residual Network; RMSE, Root Mean Squared Error; RPN, Region Proposal Network; SATD, Sum of Absolute Transformed Difference; SIFT, Scale Invariant Feature Transform; SUR, Systematic Uniform Random; SURF, Speeded-Up Robust Features; SVM, Support Vector Machines; UAS, Unmanned Aerial Systems; UAV, Unmanned Aerial Vehicle; UGV, Unmanned Ground Vehicle; VGG16, Visual Geometry Group16; VGG19, Visual Geometry Group19; YOLO, You Only Look Once; ZFNet, Zeiler and Fergus Network.
} 


\section{INTRODUCTION}

Sustainable agriculture is required to fulfill the growing population's needs by properly utilizing the available resources (Kamilaris and Prenafeta-Boldu, 2018). It can be obtained by Precision Agriculture (PA), which is supported by advanced sensing and image processing systems (Gongal et al., 2015), Artificial Intelligence (AI), etc. PA was developed in the early 1980s (Stafford, 2000). By combining modern machine vision with Deep Learning (DL) architectures, PA gains a revolutionary impact in various agricultural applications, such as crop monitoring, disease detection, and intelligent yield estimation. Among these, intelligent fruit yield estimation plays a vital role in making the final decisions regarding harvesting and fruit management.

\section{Limitations of Manual Yield Estimation}

In most countries, fruit cultivation is practiced in a large area to fulfill the worldwide demand. Therefore, improved fruit yield estimation for large orchards is required to obtain per acre fruit production and average fruit size. It enables further activities (i.e., marketing, harvesting, stock volumes, etc.) that can be planned in an effective manner by the farmers. Traditionally, fruit yield estimation has been performed by manual counting (by agricultural scientists) and leads to low precision results, high costs, as it requires expert observation, and higher time requirements for estimation. Therefore, subsequent decision making becomes a challenging task for the farmers with manual yield estimation. Hence, there is a need for an intelligent yield estimation system that overcomes the above-mentioned problems. Recently, AI-based intelligent systems for estimating fruit yield tend to provide promising results, so the problems occurring in traditional yield estimation can thereby be avoided. Further, it allows for digital agricultural systems. Machine Learning (ML) and DL are the two important techniques used in AI systems, which produces promising results in field conditions (Gongal et al., 2015; Kamilaris and Prenafeta-Boldu, 2018).

\section{Fruit Yield Estimation Using ML Techniques}

Before the ML era, fruit detection was done by simply capturing images from orchards and detecting the prominent features from the images such as size, shape, color, and texture of the fruit using various segmentation algorithms such as K-means, watershed, contour detection, and decision trees. ML is a subfield of AI and popularly used by many researchers, as it replaces the effort imparted by human intelligence. It works with a set of algorithms and develops a trained model for (given) input features obtained from source objects (Kamilaris et al., 2017; Liakos et al., 2018). The model is then used to test real-time data, which is not trained. In PA, ML is one of the most widely used techniques for decision making related to yield estimation, soil management, plant disease management, etc. Over the past decade, many works (Payne et al., 2013; Yamamoto et al., 2014; Dorj et al., 2017; Qureshi et al., 2017) have been done in fruit yield estimation using ML techniques because of its promising capability. Payne et al. (2013) proposed a method to segment mango fruit pixels based on the color components RGB and YCbCr with texture segmentation by identifying the adjacent pixels. The results showed a squared correlation coefficient $\mathrm{R}^{2}$ of 0.91 when imaging on four sides and 0.74 for one side imaging. Dorj et al. (2017) developed a method to detect and segment the citrus fruits using a watershed algorithm after converting the RGB images into an HSV color space and obtained a squared correlation coefficient $\mathrm{R}^{2}$ value of 0.93 . Some works (Stajnko and Cmelik, 2005; Malik et al., 2016; Mehta et al., 2017) adapted the size as a criterion to identify the object boundary. Even though the results are promising, these methods do not work in challenging situations such as occlusion, overlapping, and illumination variations.

Qureshi et al. (2017) proposed a method for the precise detection of fruits by analyzing images of mango tree canopies. The authors applied two approaches: The first approach dealt with identifying fruit and non-fruit pixels by applying a set of filters on the input image. The second analyzed the boundaries of mango fruits as an ellipse rather than a circular shape. Results were compared against existing ML algorithms, i.e., K-nearest neighbors (kNN) and Support Vector Machines (SVM), and the proposed method demonstrated an F1 score of 0.68. Yasar and Akdemir (2017) developed a method for orange detection using Artificial Neural Networks (ANNs) by extracting the color features obtained from an HSV color space. The detection accuracy for the test set was $89.80 \%$. Another work proposed by Zhao et al. (2016) detected the fruit pixels of immature green citrus using Sum of Absolute Transformed Difference (SATD) method. Finally, SVM classifier was employed to eliminate the false positives (based on textural features) and obtained the precision and recall values of 0.88 and 0.80 , respectively.

\section{Limitations of ML Techniques}

Even though ML techniques perform well in most fruit detection tasks, they show poor results while performing yield estimation over a large area. Because the techniques struggle to fit the model due to poor generalization capability (Yamamoto et al., 2014; Zhao et al., 2016). DL is a recently developed neural-networkbased hierarchical technique that provides promising results in almost all sectors of agriculture (Kamilaris and Prenafeta-Boldu, 2018). Intelligent fruit yield estimation using DL is an important applications of PA, which reduces the human effort considerably, as it provides high precision results and hence improved product (i.e., fruit) management (Koirala et al., 2019).

\section{Fruit Yield Estimation Using DL Techniques}

DL is a hierarchical architecture as well as a self-feature learning technique, as the layers automatically learn the features (on its own) from the raw input data (i.e., images) and hence it is more advantageous than the ML techniques. In all ML techniques, before training, the features need to be extracted from the raw input data, which is tedious and time-consuming work (Kamilaris and Prenafeta-Boldu, 2018). The Convolutional Neural Network (CNN) is one of the most widely used 
architectures in DL for various image recognition tasks, i.e., face recognition, tumor detection, weed detection, etc., as it is capable of handling the input image data by exploiting the spatial and temporal correlation, in a superior way. The general architecture of the $\mathrm{CNN}$ is shown in Figure 1. The basic CNN consists of a stack of convolutional layers, activation functions, and pooling layers. The convolutional layer is the primary layer in the $\mathrm{CNN}$ that performs convolution (i.e., element wise dot product) between the values of the input data and the kernel. The result is passed through the activation function in which a non-linearity operation is performed. To reduce the computational complexity, the features are then passed through the pooling layers for down sampling. Finally, at least one fully connected layer is used to provide the dense feature map, followed by the softmax layer in which, based on probability values, the image is classified into a particular class (Lecun et al., 2010). Starting with LeNet (Lecun et al., 1999), various CNN architectures were developed over the past decade, such as AlexNet (Krizhevsky et al., 2012), Visual Geometry Group16 (VGG16), VGG19 (Simonyan and Zisserman, 2015), ResNet (He et al., 2015), GoogLeNet (Szegedy et al., 2015), DenseNet (Huang et al., 2016), and SqueezeNet (Iandola et al., 2016). Each architecture differs in terms of the number of convolutional layers, non-linearity functions, type of pooling operation used, etc.

In recent years, the $\mathrm{CNN}$ has been used for immense applications in the agricultural sector such as disease detection and prediction of soil organic matter (Xu et al., 2019). Torres et al. (2020) reviewed the various CNN architectures, namely AlexNet, VGG16, and GoogLeNet, for fruit detection, classification, sorting, and quality control tasks. They concluded that, if the task is more complex, the kernels as well as the number of layers need to be increased for improved feature extraction. Wang and Chen (2020) proposed a method to categorize the fruits by using a deep CNN. Their architecture consists of 8 layers of CNNs and provided an overall accuracy of $95.67 \%$. The modification in the architecture is that the authors used a non-linearity function of a parametric Rectified Linear Unit (ReLU) instead of a plain ReLU, and a dropout layer is added before each fully connected layer. It is important to note that any success of the DL architecture depends on the large amount of training data, as it is a data hungry architecture. However, collecting and labeling more training images is tedious work. Hence, Rahnemoonfar and Sheppard (2017) proposed a simulated learning method, in which training was performed using synthetic images, and testing was done on real-time data. The authors used a modified ResNet-Inception model to train the synthetic images, and the results portrayed training and testing accuracies of 93 and $91 \%$, respectively.

\section{Limitations of DL Techniques}

Various research studies have been performed for fruit yield estimation using DL (Wang et al., 2013; Bargoti and Underwood, 2017; Rahnemoonfar and Sheppard, 2017; Sun et al., 2018; Torres et al., 2020). In general, DL techniques perform well for most fruit detection tasks. However, one of the issues associated with the DL technique is a lower spatial resolution followed by pooling operation, which results in a poor localization of objects present in a particular scene. The exact location of the fruit needs to be localized for improved prediction. Hence, in order to obtain good accuracy in the process of automatic fruit yield estimation, DLbased semantic segmentation architectures are now employed widely (Tu et al., 2018).

This review paper is organized as follows: section "Intelligent Fruit Yield Estimation" describes fruit yield estimation employing DL-based semantic segmentation, which includes tree sampling, different sensing technologies, data augmentation methods, and different semantic segmentation architectures. The various challenges that occur when developing an intelligent fruit yield estimation system are discussed in section "Challenging Issues in Intelligent Fruit Yield Estimation System." Section Conclusion concludes the paper.

\section{INTELLIGENT FRUIT YIELD ESTIMATION}

The different steps involved in developing an intelligent yield estimation system are tree sampling, data capturing using different sensing technologies, data augmentation, fruit detection, counting and yield estimation using DLbased semantic segmentation architectures, and performance evaluation (as shown in Figure 2).
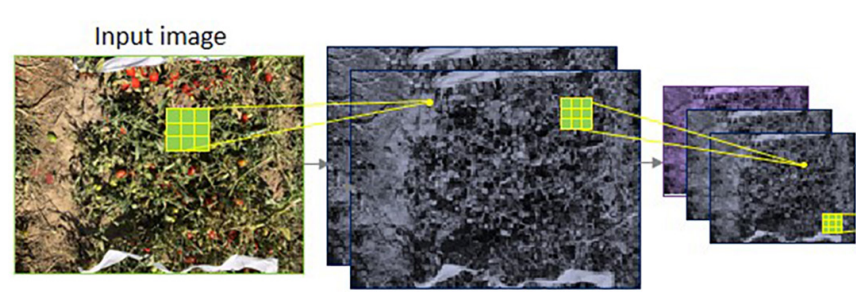

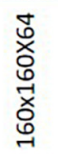

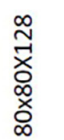

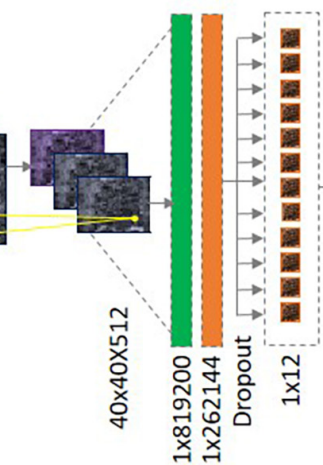

Probability of each class

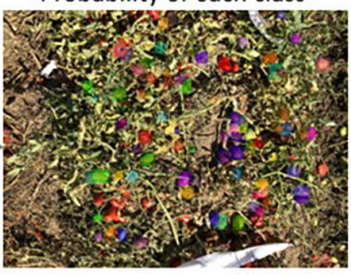

Legend

Dense $\square$ convolution

Flaten Maxpooling

FIGURE 1 | Illustration of a typical Convolutional Neural Network (CNN). 

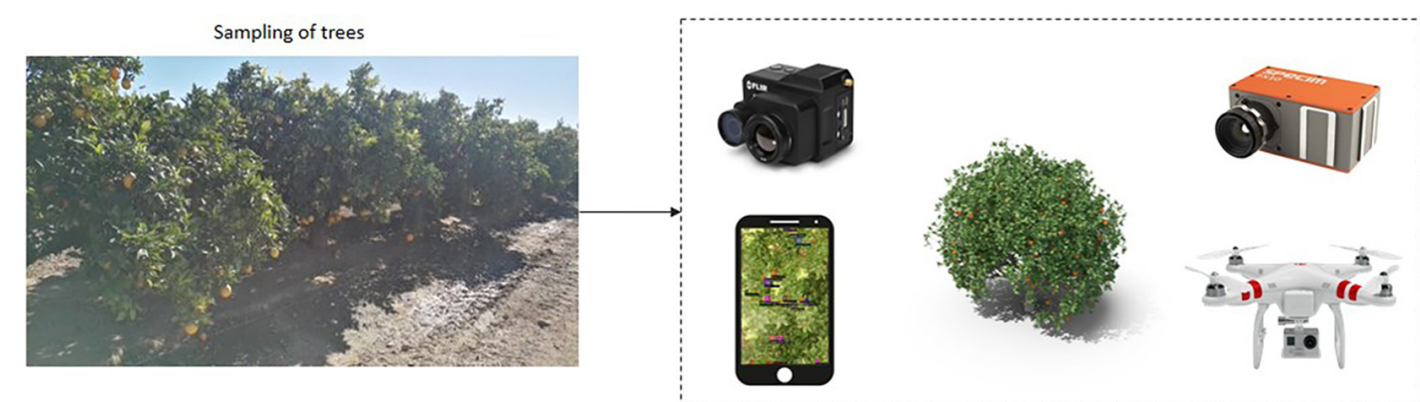

Data capturing by different sensors

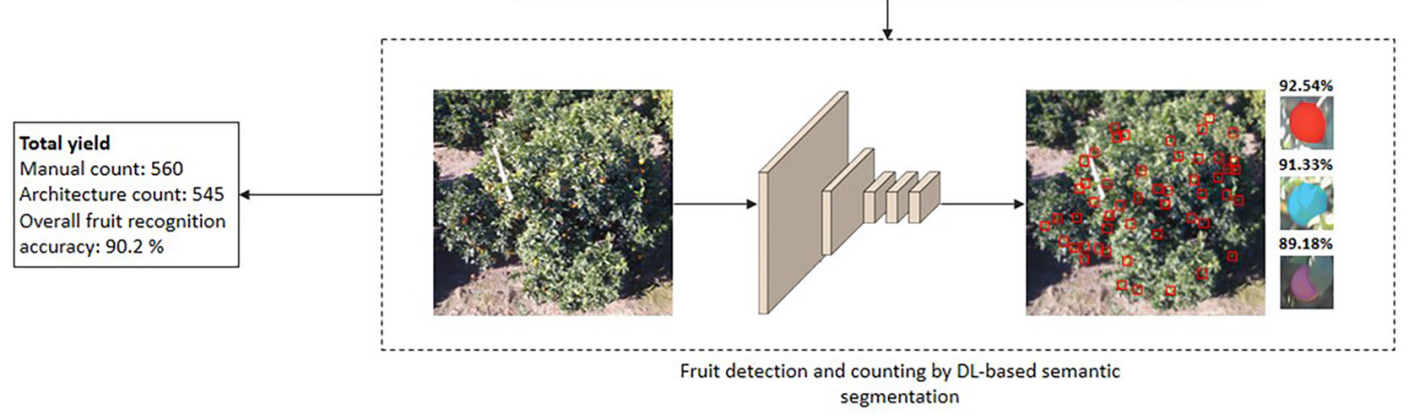

FIGURE 2 | Intelligent fruit yield estimation in orchards.

\section{Tree Sampling}

Before collecting images from the orchards, the initial step is to sample the trees, which decides the number of representative trees to be taken over the entire population. The sample selection must be sufficient: it ultimately represents the whole orchard's population, and a perfect yield can thus be estimated. Based on the survey theory, two approaches are primarily used for sampling, namely design-based and model-based approaches. For diverse populations, the design-based approach is adapted for sampling, whereas the model-based approach performs well in systems which described by the spatial positioning of a population. Various sampling procedures for designbased and model-based approaches include simple random sampling, systemic sampling, a smooth fractionator, probability proportional to size sampling, and stratified sampling, etc. (Cochran, 1977; Wulfsohn et al., 2010).

A comparative study proposed by Uribeetxebarria et al. (2018) compares the sampling efficiency in yield estimation of fruit orchards using simple random sampling and stratified sampling. The authors used the Normalized Difference Vegetation Index (NDVI) and the apparent Electrical Conductivity (ECa) for stratified sampling. As a result, the plot's sampling size was reduced by $17 \%$ compared with simple random sampling, which improves the precision of fruit yield estimation. Table 1 shows the various sampling methods to sample the trees in an orchard.

\section{Data Capturing Using Different Sensing Technology}

The primary system for fruit detection is the sensing system. It should capture the focused images in field conditions by tackling challenging situations such as variable lighting conditions and resolution. Figure 3 shows the different camera models presently available in the market with various features (i.e., black and white, RGB, thermal, etc.).

Earlier studies were performed to detect fruits in orchards using black and white cameras. Without color, the widespread availability of features was exploited to detect the fruits in the canopy. After the color sensors, RGB cameras were mostly used in all detection systems (to capture the color), which made the detection process easier. Complementary Metal-OxideSemiconductors (CMOSs) and Charge Coupled Devices (CCDs) are prevalent technologies used as color sensors and are used widely in all machine vision systems. CCD sensors operate by capturing the entire frame simultaneously, whereas a CMOS captures the images pixel by pixel. Thermal imaging has also been used in some fruit detection works (Gongal et al., 2015). Here, each object's feature (i.e., branch, stem, etc.) is detected based on temperature, as fruits have a higher temperature than the background objects. Spectral imaging is a next-generation camera model (i.e., multispectral and hyperspectral sensors) currently used. It gives additional information related to spectral details (at each color space) along with color features. Even if the fruit color and background (such as the leaves and stem) are the same, fruit detection can be performed using spectral information (Feng et al., 2019).

Multispectral images have fewer broader spectral bands (3-15), whereas hyperspectral images have a greater number of narrower spectral bands (20-250). The Landsat-8 satellite is an example of a multispectral imager consisting of 11 bands with a high spatial resolution of $30 \mathrm{~m}$ in most bands. NASA's Airborne Visible/Infrared Imaging Spectrometer (AVIRIS) is a hyperspectral imager with 224 bands with $0.4-2.5 \mu \mathrm{m}$ (Kwan, 2019). 
TABLE 1 | Various sampling methods for tree sampling in an orchard.

\begin{tabular}{|c|c|c|c|}
\hline Sampling techniques & Description & Merits & Demerits \\
\hline $\begin{array}{l}\text { Simple random sampling } \\
\text { (Cochran, 1977) }\end{array}$ & $\begin{array}{l}\text { Randomly selecting samples } \\
\text { from the whole population. }\end{array}$ & $\begin{array}{l}\text { Completely represent the } \\
\text { entire population. }\end{array}$ & $\begin{array}{l}\text { Expensive and } \\
\text { time-consuming. }\end{array}$ \\
\hline $\begin{array}{l}\text { Systematic random } \\
\text { sampling (Mostafa and } \\
\text { Ahamad, 2018) }\end{array}$ & $\begin{array}{l}\text { Provides an improved tradeoff } \\
\text { between the precision of the } \\
\text { estimator and the sampling } \\
\text { interval. }\end{array}$ & $\begin{array}{l}\text { Faster than simple random } \\
\text { sampling. }\end{array}$ & $\begin{array}{l}\text { Realization is difficult } \\
\text { without knowing all the } \\
\text { members in the population. }\end{array}$ \\
\hline $\begin{array}{l}\text { Stratified sampling } \\
\text { (Uribeetxebarria et al., } \\
\text { 2018) }\end{array}$ & $\begin{array}{l}\text { From the whole population, } \\
\text { strata or sub categories are } \\
\text { considered. Samples are taken } \\
\text { from these strata randomly. }\end{array}$ & $\begin{array}{l}\text { High precision and requires } \\
\text { smaller samples. }\end{array}$ & $\begin{array}{l}\text { If the sub category is not } \\
\text { properly chosen, it is } \\
\text { challenging to represent the } \\
\text { entire population. }\end{array}$ \\
\hline $\begin{array}{l}\text { Smooth fractionator } \\
\text { (Gundersen, 2002) }\end{array}$ & $\begin{array}{l}\text { Systemic sampling is applied to } \\
\text { each uniquely (based on shape, } \\
\text { size, texture, etc.) divided unit } \\
\text { from the whole population for } \\
\text { efficient sampling. }\end{array}$ & $\begin{array}{l}\text { Robust for the } \\
\text { heterogeneous population. }\end{array}$ & $\begin{array}{l}\text { When the population of } \\
\text { interest is sparsely } \\
\text { distributed, it is inefficient. }\end{array}$ \\
\hline $\begin{array}{l}\text { Cluster sampling (Hamilton } \\
\text { and Hepworth, 2004) }\end{array}$ & $\begin{array}{l}\text { Suitable for large and complex } \\
\text { populations. }\end{array}$ & $\begin{array}{l}\text { Minimum resources for the } \\
\text { sampling process. }\end{array}$ & High sampling error. \\
\hline $\begin{array}{l}\text { Multistage sampling } \\
\text { (Chauvet, 2015) }\end{array}$ & $\begin{array}{l}\text { At various levels, sampling is } \\
\text { performed. }\end{array}$ & More flexible. & $\begin{array}{l}\text { Large number of errors due } \\
\text { to clusters in different } \\
\text { stages. }\end{array}$ \\
\hline $\begin{array}{l}\text { Probability proportional to } \\
\text { size sampling (Gardi et al., } \\
\text { 2008) }\end{array}$ & $\begin{array}{l}\text { Each population has a size } \\
\text { before sampling, which is } \\
\text { proportional to the probability of } \\
\text { selecting a unit. }\end{array}$ & $\begin{array}{l}\text { Well suited for sparsely } \\
\text { distributed populations. }\end{array}$ & $\begin{array}{l}\text { Reduced precision when } \\
\text { sampling more clustered } \\
\text { units. }\end{array}$ \\
\hline
\end{tabular}

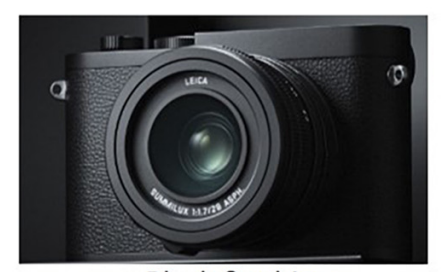

Black \& white camera (Leica Q2) www.leica-store.in

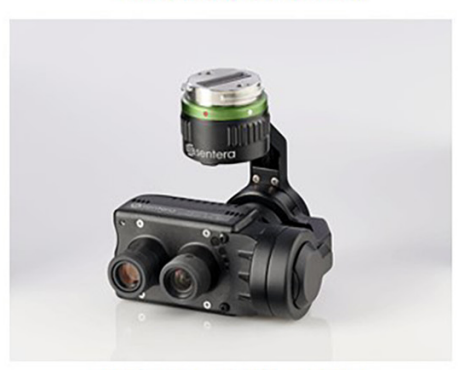

Multispectral camera

(AGX710)

www.sentera.com

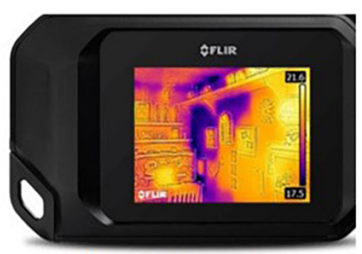

Thermal camera

(Flir c2)

www.digikey.in

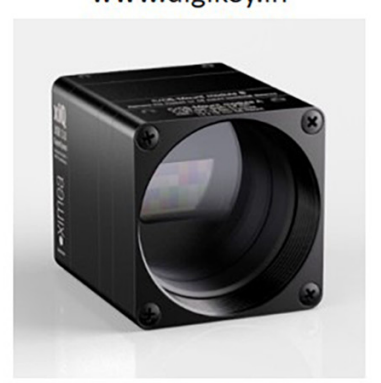

Hyperspectral camera

(MC124MG-SY)

www.ximea.com

FIGURE 3 | Different types of camera models using various sensor technologies.

Bulanon et al. (2008) explored a method for citrus fruit detection by capturing images using a thermal camera. The temperature gradient was calculated based on the emissivity of the fruit, relative humidity, and ambient temperature from the captured thermal images. Finally, these data were used to segment the fruits from the background using image processing algorithms. The results showed that the average true positive and false positive were 0.70 and 0.06 , respectively.

Gan et al. (2018) developed a method for fruit detection based on multi-modal imaging i.e., combining both color and thermal images using a color thermal combined probability algorithm. It effectively extracts the information present in both images. As a 
result, the precision and recall rate were improved for detecting the immature green citrus fruits. Recall and precision were 78 and $86.60 \%$, respectively, with only color images and 90.40 and $95.50 \%$, respectively, with multimodal imaging.

Feng et al. (2019) proposed a method for detecting the apples using multispectral dynamic imaging. Using this, the pictures were taken at a considerably high level of contrast between background and fruit, which improves the recognition accuracy as 92\%. Okamoto and Lee (2009) developed a method for identifying the green citrus fruit using hyperspectral imaging. The authors used the spectral wavelength of $369-1042 \mathrm{~nm}$ for capturing pictures and obtained a detection accuracy of $70-85 \%$. Table 2 describes the various cameras available in the market for capturing pictures along with their resolution. The next step is the preprocessing of images, which includes data augmentation and resizing.

\section{Data Augmentation}

Data augmentation is a useful technique in neural-network-based systems, as DL deals with a large amount of data. Practically, it is complicated to collect and annotate such a large volume of the dataset for training. However, the small dataset will lead to an over-fitting problem, and hence the system will work well in the training phase and will not produce accurate results during the testing phase. Therefore, in order to increase the available dataset to a large number, various transformations will be performed through data augmentation steps, such as translation, rotation, adding noise, cropping, and flipping. It will improve the network capability to learn the deep features present in the data (Shorten and Khoshgoftaar, 2019; Zheng et al., 2020). Figure 4 shows some of the transformations applied to a captured image for a (sample) mango tree from an orchard.

Kestur et al. (2019) proposed a method for estimating the yield of mango fruit. The authors collected 40 images with a size of $4,000 \times 3,000$, and it is complicated to process the same with the original size. Hence, they used the cropping augmentation method to generate the image patches with a size of $200 \times 200$. After augmentation, the training and testing dataset had 11,096 and 1,500 patches, respectively. The final result showed an F1 score of 0.844 for the developed architecture with data augmentation.

Stein et al. (2016), proposed a method for detecting the mango fruit using anFaster R-CNN method. In this work, the total dataset consists of 71,609 mangoes from 522 trees. They performed the augmentation by randomly sampling the subset of images from the original dense dataset, thereby capturing the entire orchard block's variability. Image using augmentation helps to overcome the memory constraints required for processing the images. They achieved an F1 score of 0.881 in the test images.

\section{Implementation Using DL-Based Semantic Segmentation Techniques}

For dense pixel-wise prediction, semantic segmentation is the best choice, and DL is most suitable for the hierarchical learning of data. In order to exploit its full potential, recent researchers have combined semantic segmentation with DL techniques, resulting in the highest precision accuracy, especially in the domain of fruit yield estimation. Combining semantic segmentation with DL architectures, the main objective of recent research is to obtain a perfect counting of fruits, which reveals the accurate yield for a specified orchard in challenging situations such as occlusion, overlapping and illumination variations (Payne et al., 2016; Sun et al., 2018; Guo et al., 2019).

Basically, fruit is detected by capturing image data and transforming it into a more detailed feature space that details every pixel present in the image. The overall DL-based semantic segmentation architectures are divided into three groups, namely a CNN with pixel-based prediction, fully convolution prediction, and region-based prediction. The first group obtains the input as an image patch and predicts each pixel into a particular class using score vectors. The second group processes the whole image, and prediction is performed based on score maps. In the third group, regions are extracted from the input image, and these

TABLE 2 | Different types of camera models available on the market.

\begin{tabular}{|c|c|c|c|c|}
\hline Sensors & Model & Resolution & Sensor size & References \\
\hline \multirow[t]{3}{*}{ Black and white sensor } & Leica Q2 & $8,368 \times 5,584$ & $36 \times 24 \mathrm{~mm}$ & www.leica-store.in \\
\hline & Nikon z7 & $3,840 \times 2,160$ & $35.9 \times 23.9 \mathrm{~mm}$ & www.nikon.co.in \\
\hline & Canon EOS 5D & $4,368 \times 2,912$ & $36 \times 24 \mathrm{~mm}$ & www.canon-europe.com \\
\hline \multirow[t]{3}{*}{ RGB sensor } & Sony a5100 & $6,000 \times 4,000$ & $23.5 \times 15.6 \mathrm{~mm}$ & www.sony.co.in/ \\
\hline & Ricoh GR III & $6,000 \times 4,000$ & $23.5 \times 15.6 \mathrm{~mm}$ & www.ricoh-imaging.co.jp \\
\hline & Fujifilm X-E3 & $6,000 \times 4,000$ & $23.5 \times 15.6 \mathrm{~mm}$ & www.fujifilm-x.com \\
\hline \multirow[t]{3}{*}{ Thermal sensor } & Flir c2 & $320 \times 240$ & $128 \times 96 \mathrm{~mm}$ & www.digikey.in \\
\hline & Testo 871 & $320 \times 240$ & Not available & www.testo.com \\
\hline & Fluke Tl450 & $320 \times 240$ & Not available & www.flukeindia.com \\
\hline \multirow[t]{3}{*}{ Multispectral sensor } & AGX710 & 12.3 MP & $89 \times 88 \times 98 \mathrm{~mm}$ & www.sentera.com \\
\hline & MSC-AGRI-1-A & $512 \times 512$ & $5.5 \times 5.5 \mu \mathrm{m}$ & www.spectraldevices.com \\
\hline & MSC-RGBN-1-A & $512 \times 512$ & $5.5 \times 5.5 \mu \mathrm{m}$ & www.spectraldevices.com \\
\hline \multirow[t]{3}{*}{ Hyperspectral sensor } & MC124MG-SY & $4,112 \times 3,008$ & $14.2 \times 10.4 \mathrm{~mm}$ & www.ximea.com \\
\hline & MQ022HG & $2,048 \times 1,088$ & $11.3 \times 6.0 \mathrm{~mm}$ & www.ximea.com \\
\hline & OCI-UAV-1000 & 2048 & Not available & www.bayspec.com \\
\hline
\end{tabular}


A Translation

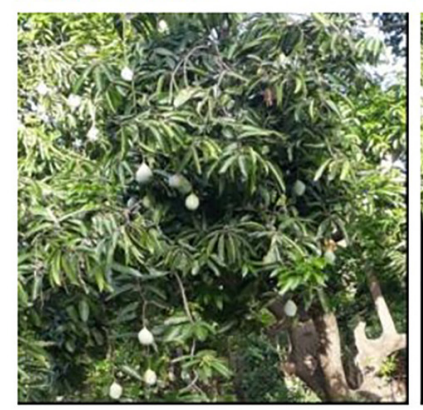

B Rotation with $10^{\circ}$

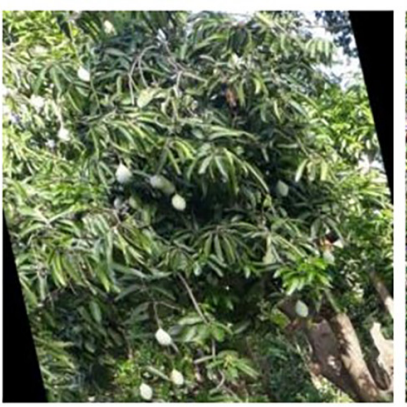

C Adding noise

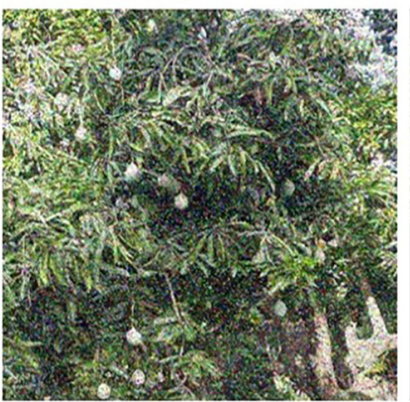

D Rotation with $20^{\circ}$

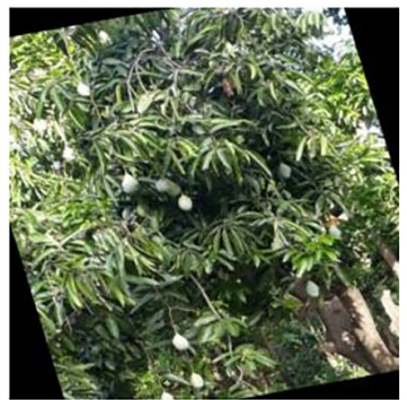

FIGURE 4 | Random transformations intended for data augmentation.

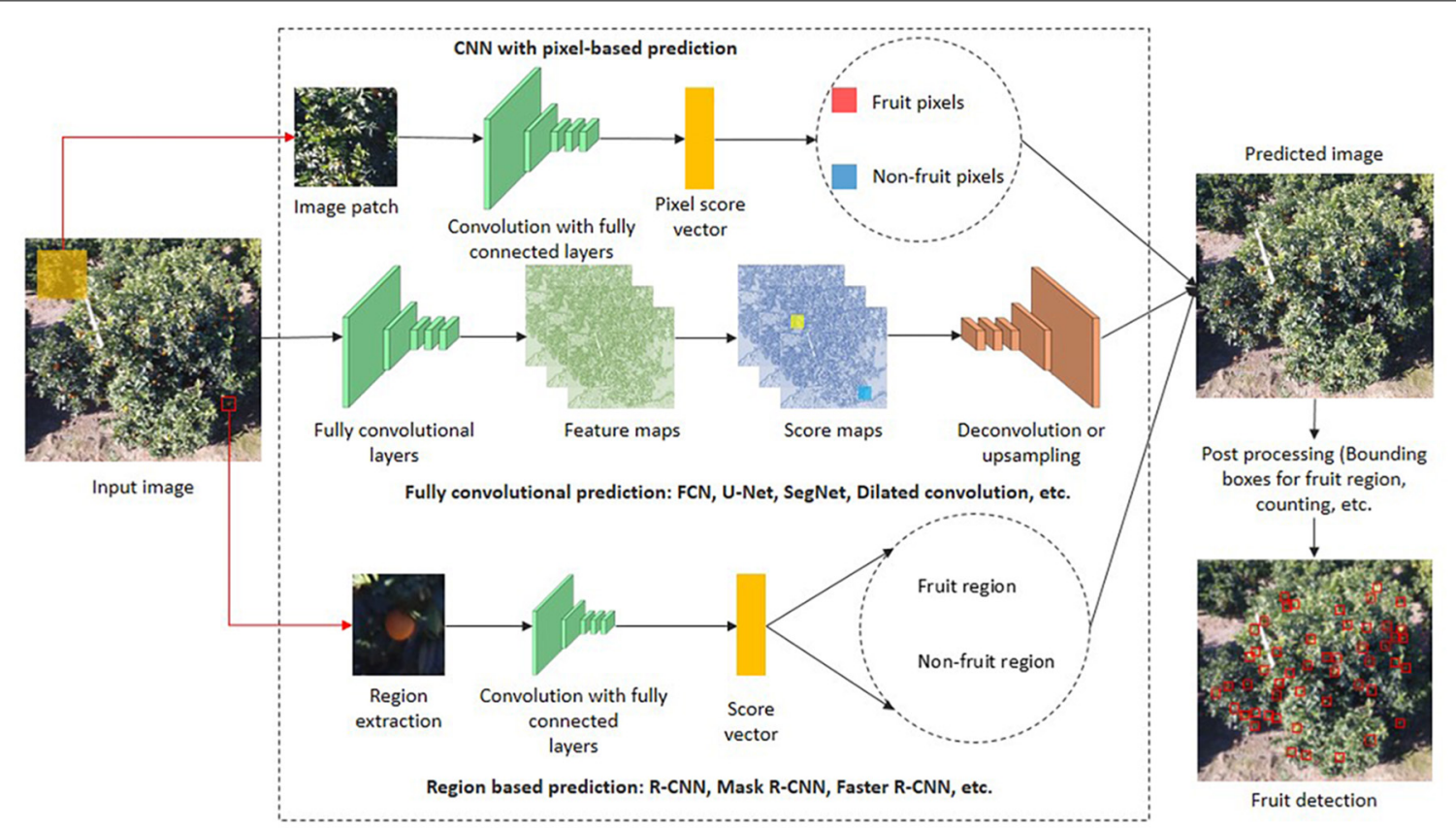

FIGURE 5 | Fruit detection and localization using various DL-based semantic segmentation techniques.

regions are the input for the architectures. Based on the score vector, each region is labeled as a fruit or non-fruit region. The architectures present in each group is explained in section "Popular Semantic Segmentation Architectures in Fruit Yield Estimation." Post-processing is then applied to the segmented pixels to group the adjacent pixels to detect the whole fruit present in a particular image. An entire process of DL-based semantic segmentation for fruit detection is depicted in Figure 5.

\section{Popular Semantic Segmentation Architectures in Fruit Yield Estimation}

Semantic segmentation provides a complete understanding of a particular scene by labeling each pixel of an image to a specific class. It is one of the essential techniques presently used in almost such fields as agriculture, medicine, and autonomous navigation. It plays an inevitable role in object detection and localization tasks (for e.g., fruit detection in the orchards). Initially, semantic segmentation was performed using various graphical approaches such as super pixels segmentation, Markov Random Fields (MRFs), forest-based methods, Conditional Random Fields (CRFs), and dense CRFs. These methods tend to find the correlations between the adjacent pixels and obtain an inferences from them. Each pixel was labeled to a specific class based on the inference obtained from the above-mentioned graphical approaches (Shi and Malik, 2000; Ren and Malik, 2003; Silberman and Fergus, 2011; Nematollahi and Zhang, 2014; Khan et al., 2015; Yu et al., 2018).

For an improved representation of objects, the features of all objects (present in an image) need to be distinguished clearly. In this regard, hand-engineered features such as Speeded-Up Robust Features (SURF) (Woods et al., 2019), Histograms of 
Oriented Gradient (HOGs) (Tan et al., 2018), and Scale Invariant Feature Transform (SIFT) (Tu et al., 2018) were used to obtain reasonable features from a given image. However, in these methods, useful features need to be identified, which is a tedious process. Results of object recognition highly depend on extracted features; otherwise, the system will fail to give accurate results. For a large amount of data, the above said methods struggle to obtain complex data features.

Since the development of DL, hierarchical features have been learned from the source objects directly with hence no need for the above-mentioned hand-engineered feature learning methods. The pre-trained architectures described in section "Fruit Yield Estimation Using DL Techniques" are well suited for object detection tasks. The disadvantage is that these pre-trained architectures have suffered due to computational complexity and pooling operations. The fully connected layers present in CNNs create computational complexity, and spatial resolution has been lost due to pooling operations. Therefore, an improved localization of objects could not be achieved. Hence, DL-based semantic segmentation architectures were developed to obtain dense pixel-based prediction and improved feature learning strategies. As a result, various architectures have been explored for semantic segmentation by modifying and finetuning DL's pre-trained models, namely, a CNN with pixel-based classification, FCN, SegNet, Dilated convolution, PSP Net, and weakly supervised learning models. These architectures provide perfect labeling to the raw input data and better detect all objects in a particular scene (Long et al., 2014; Badrinarayanan et al., 2017; Luo et al., 2017; Ulku and Akagunduz, 2019; Liu et al., 2019).

\section{CNN With Pixel-Based Prediction}

In this method, image patches with a fixed size centered at each pixel are given to the $\mathrm{CNN}$. In each image patch, the pixel labeling of a small region is not enough to make a localizationbased decision. To overcome this issue, image patch size can be increased at the cost of more parameters calculations and hence creates computational complexity (Yu et al., 2018). This inefficient way of computation has been overcome by fully convolution prediction.
Kestur et al. (2019) proposed MangoNet, an architecture that detects and counts the fruits in an open orchard using pixel-based semantic segmentation. The original images collected from the orchard were converted into $200 \times 200$ image patches, and a totally 11,096 images were given to the MangoNet for training. After training, the architecture was tested with 1,500 image patches of $200 \times 200$ pixels from four test images. The proposed MangoNet achieved a pixel accuracy of $73.60 \%$ and an F1 score of 0.84 . Table 3 shows some of the literature related to CNNs with pixel-wise prediction.

\section{Fully Convolutional Prediction Fully Convolutional Networks (FCN)}

The first semantic segmentation architecture using a deep CNN was FCN (Long et al., 2014). Here, there are no fully connected layers; instead, it only has convolutional layers. The pre-trained architectures such as AlexNet, VGG16, and GoogLeNet, etc., were transformed into a new semantic segmentation architecture by fine tuning the fully connected layers. The FCN architecture is shown in Figure 6. The idea behind the FCN is that creating segmentation maps for the images of different resolutions and hence the localization of objects can be achieved by retaining the spatial resolution.

Liu et al. (2018) proposed a novel approach that uses one of the semantic segmentation FCN architectures to segment fruits from video frames. After segmentation, tracking was performed using a Hungarian algorithm with a cost function defined by a Kalman filter. Two fruit datasets (oranges and apples) with different features in the sense of variations in depth and illumination, the resemblance of color between foliage and fruit, and occlusion were used to evaluate the proposed method. An error mean of 0.20 and $3.30 \%$, a standard deviation of 7.80 and $4.10 \%$ and a L1 error of 203 and 322 were obtained for the orange and apple datasets, respectively.

Lin et al. (2019) developed a method to detect the guava fruit using the FCN technique. The authors performed fine tuning in the original FCN architecture by employing bilinear interpolation and Adam optimizer with a learning rate of 0.0001. The trained FCN architecture was used to detect the guava fruit in the field. The proposed method's detection

TABLE 3 | CNN with pixel-based prediction literature.

\begin{tabular}{|c|c|c|c|}
\hline Methodology & Authors and year & Dataset & Results \\
\hline $\begin{array}{l}\text { Apple detection and yield } \\
\text { estimation using } \\
\text { multilayered perceptron } \\
\text { and CNN }\end{array}$ & Bargoti and Underwood, 2017 & $\begin{array}{l}8,000 \text { images of } 1,232 \times \\
1,616 \text { pixel, each } \\
32 \text { sub images of } 308 \times \\
202 \text { pixels obtained from } \\
\text { each image }\end{array}$ & $\begin{array}{l}\text { F1 score was } 0.791 \text {, and detection } \\
\text { F1 score was } 0.861 \text {. } \\
\text { Squared correlation coefficient } R^{2} \\
\text { was } 0.826 \text {. }\end{array}$ \\
\hline $\begin{array}{l}\text { Mango fruit detection and } \\
\text { localization using multiple } \\
\text { view geometry }\end{array}$ & Stein et al., 2016 & $\begin{array}{l}71,609 \text { mangoes scanned } \\
\text { from } 522 \text { trees }\end{array}$ & $\begin{array}{l}\text { Single view squared correlation } \\
\text { coefficient } R^{2} \text { was } 0.81 \text {, dual view } \\
\text { and multi view } R^{2} \text { was } \geq 0.90 \text {. }\end{array}$ \\
\hline $\begin{array}{l}\text { Apple yield estimation using } \\
\text { multi-scale sparse auto } \\
\text { encoder feature learning } \\
\text { method }\end{array}$ & Hung et al., 2015 & $\begin{array}{l}8,000 \text { apple images of the } \\
\text { dataset } \\
\text { Image size of } 1,232 \times \\
1,616 \text { pixels }\end{array}$ & $\begin{array}{l}\text { Squared correlation coefficient, } \mathrm{R}^{2} \\
\text { was } 0.81 \text {. } \\
\text { Global accuracy was } 92.5 \% \text {, } \\
\text { average accuracy was } 85.1 \% \text {, and } \\
\text { F1 score was } 87.3 \% \text {. }\end{array}$ \\
\hline
\end{tabular}




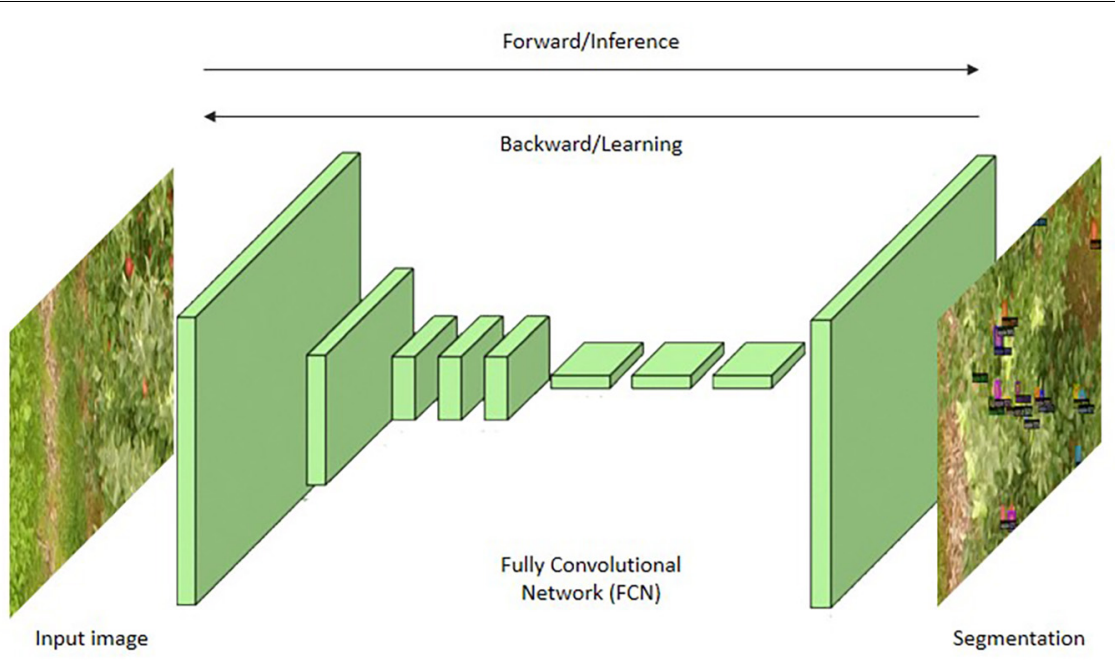

FIGURE 6 | Fully Convolutional Network (FCN).

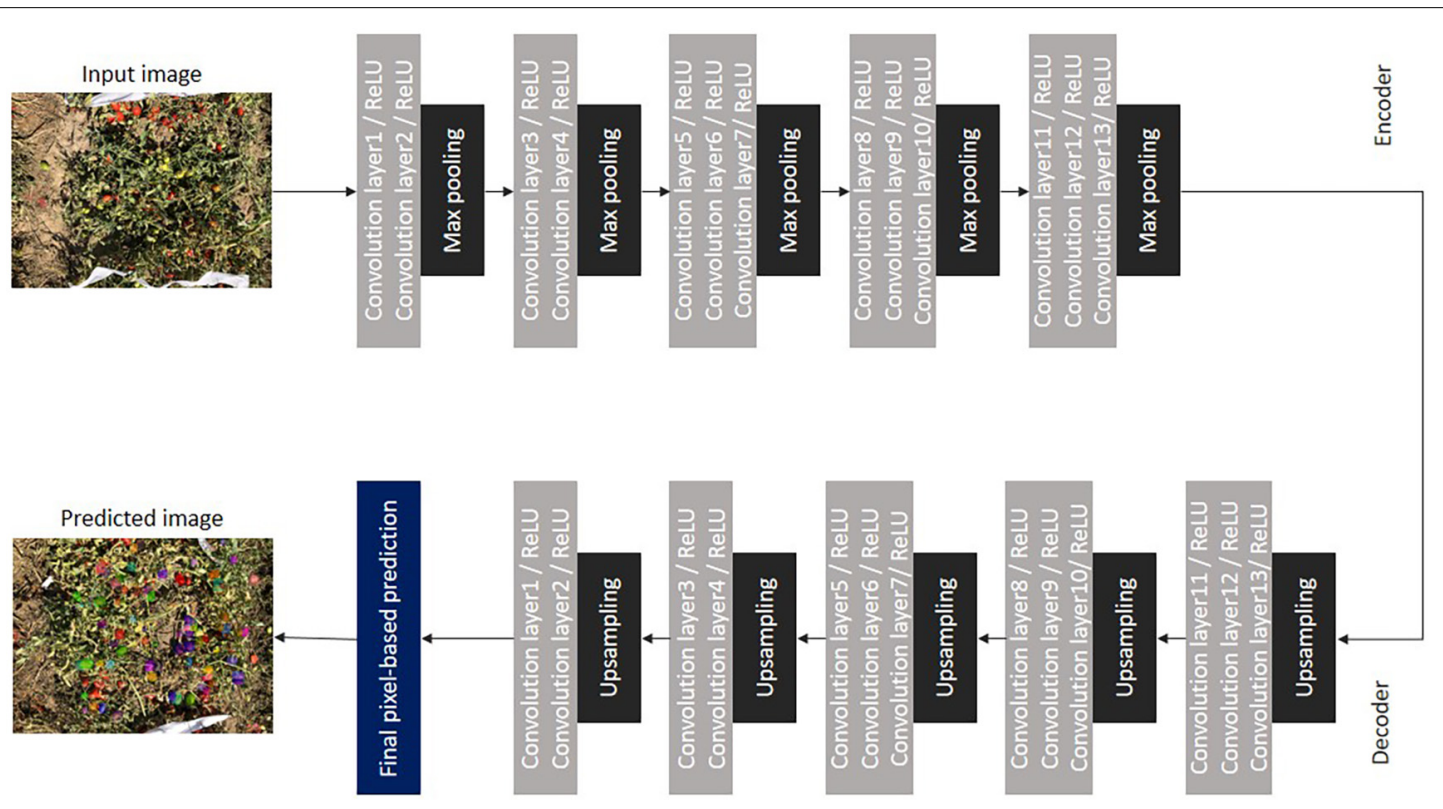

FIGURE 7 | Encoder-decoder architecture of SegNet.

accuracy in terms of precision and recall was 0.983 and 0.948 , respectively.

\section{Encoder-Decoder Architecture}

In addition to the FCN, the encoder-decoder architecture was introduced. SegNet is one of the most popular encoder-decoder architectures that implements transposed convolution.

The SegNet architecture is shown in Figure 7. For developing this network, VGG16 was modified by removing the (last) three fully connected layers and used as an encoder. The decoder consists of 13 convolutional layers in addition to upsampling layers. The purpose of upsampling layers is to obtain indices of pooling layers. Bilinear interpolation was used in the upsampling layers for obtaining the lost size of the input due to the down sampling process (Badrinarayanan et al., 2017).

Architectures similar to SegNet were developed, namely the Deconvolution Network (DeconvNet) (Noh et al., 2015) and U-Net (Ronneberger et al., 2015). DeconvNet performs semantic segmentation at the cost of high computational resources and is very hard to train, as it has more parameters in the feature maps. U-Net differs from SegNet in upsampling operations. $\mathrm{U}$-Net is designed to send the entire feature map to the decoder instead of sending the pooling indices as in SegNet, thus consuming more memory.

Hani et al. (2019) proposed a comparative study for fruit detection and yield mapping using three architectures: U-Net, 
the Gaussian Mixture Model (GMM), and the faster R-CNN. In the case of U-Net, 103 images of a $1,920 \times 1,080$ pixel size of apple trees with different varieties, tree shapes, and growing stages were used for training. The U-Net performed best and produced high recall for the given dataset. The results for fruit detection using U-Net implied that it achieves good performance when the testing dataset is similar to the training dataset, as there were poor generalization results for different testing set.

\section{Dilated Convolution and Pyramid Scene Parsing Network}

Another version of semantic segmentation architecture is dilated convolution (Chen et al., 2017), and it is also known as atrous-convolution or holes' algorithm (Holschneider et al., 1989). Its operation is simply based on an undecimated wavelet transform. Using atrous-convolution by changing the receptive field's dilation factor (Luo et al., 2017), the dense prediction was obtained in the dilated convolution. In standard convolution, the dilation rate (r) is one. By inserting zeros, the kernel size is effectively increased (with only the same non-zero values) without affecting the computation cost. Hence, the spatial resolution of the feature maps can be maintained for dense segmentation tasks.

In a deep $\mathrm{CNN}$, the context information is determined by the size of the receptive field. Ongoing through high layers, the receptive field is smaller, so the network could not incorporate the global context information. Zhao et al. (2017) addressed this problem by introducing an effective global prior representation. For obtaining discriminative features among various objects present in a particular scene, this work introduced a representative method to fuse information among different sub-regions (with these receptive fields). The pyramid scene parsing technique used the ResNet architecture with a dilated network for an effective representation of the global context present in an image.

Kang and Chen (2019) proposed a backbone network to detect and segment the apples and branches in an orchard using visual sensors attached with the robotic arm. This network used the atrous pyramid spatial pooling and Gate Feature Pyramid Network (GFPN) to improve the learning capacity, at all levels of spatial resolutions. The backbone network was based on the ResNet101 architecture, which has residual connections that extract the depth features and avoid vanishing gradient problems while passing through the back propagation stage. Three models have been developed, namely DasNet-A, DaSNet-B, and DaSNetC. The GFPN admits only the selective features as a representative (among different levels). It reduces the spatial shift, and gradient values can be balanced during the steps of back propagation.

\section{Multipath Refinement Network}

The Refinement Network (RefineNet) (Lin et al., 2017) is a multipath networks that generates a high-resolution feature maps by obtaining all informations from the down sampling process. It uses residual connections in order to obtain a high-level semantic feature map, which ensures an improved segmentation of objects. The pre-trained architectures ResNet developed by $\mathrm{He}$ et al. (2015) was used to build refinement networks. In this regard, the
ResNet is divided into four blocks, and each RefineNet block is connected to the output of the respective ResNet block. In order to obtain improved results, the developed multipath network accepts input from the multiple ResNet blocks. To the best of our knowledge, no work has yet been carried out using RefineNet, in the domain of fruit yield estimation.

\section{Region Based-Prediction Regions With Convolutional Neural Networks (R-CNN)}

In the R-CNN developed by Girshick et al. (2014) the bottomup regions are first extracted (from the input images), and these regions are the input to the $\mathrm{CNN}$ for extracting the features. Finally, linear SVMs are used to classify the pixels into a particular class, and improved detection and localization can thereby be achieved for semantic segmentation. More computation time is one of the bottlenecks of the R-CNN. Hence, researchers have explored superior methods of detecting the object, namely the faster R-CNN (Ren et al., 2016). This network has a Region Proposal Network (RPN), which consists of fully convolutional layers and provides region proposals from the input images by predicting the boundaries of an object. Object detection using the R-CNN will be performed. The extension of faster R-CNN is mask R-CNN (He et al., 2017), which has an additional unit to predict the mask of an object along with the existing bound box recognition unit. The mask R-CNN is best suited for advanced stages of segmentation, i.e., instance segmentation, where each object present in an image is detected and differentiated separately by predicting the mask (for each distinct object).

Small (passion) fruit detection and counting was performed by $\mathrm{Tu}$ et al. (2020) using a multiple-scale faster R-CNN using RGB-Depth images. Two modules were used: the RPN and the faster R-CNN. The first module was used to generate the object proposals. These object proposals were fed as inputs to the second module, which detected the fruits with bounding boxes.

Wan and Goudos (2020) proposed a method for multi-class fruit detection using an improved faster R-CNN architecture. The authors used three varieties of fruits: apple, mango, and orange. The dataset consists of 820 apple images, 822 mango images, and 799 orange images of a $100 \times 100$ pixel size. The penalty factor and iteration were chosen as 200 and 5,000, respectively. The improved faster R-CNN obtained precision values of 92.51, 88.94, and $90.73 \%$ for apple, mango, and orange fruits, respectively. The processing speed was $50 \mathrm{~ms} /$ image. Compared with other DL-based semantic segmentation architectures, such as YOLO, fast R-CNN, faster R-CNN, YOLOv2, and YOLOv3, and the proposed method outperformed than the other architectures both in precision and processing speed.

Apolo-Apolo et al. (2020) developed a model for citrus fruit detection using faster R-CNN architecture. The images from 20 sample trees were captured from the citrus orchard using a Unmanned Aerial Vehicle (UAV). Faster R-CNN is one of the pre-trained semantic segmentation architectures used for training the orange dataset. Features extracted from the images using CNN were given as input to the region proposal network. 
This network consists of stack of convolutional layers followed by non-linearity function and max pooling layers. Object proposals were identified using the CNN based on the object score obtained at each position. Then, the classification, bounding box prediction and size of the objects were estimated. The proposed model achieved more than $90 \%$ precision and an F1 score of more than $89 \%$. False positives were observed in challenging situations such as sunlight variation and immature fruits. The standard error for fruit count using the proposed model was $6.59 \%$ against the visual count. Based on the count, yield of the whole orchard was estimated using the Long Short-Term Memory (LSTM) model. It is a recurrent neural network that predicts the present data using the information obtained from the past data which helps to identify the complex pattern of the data. The real yield data collected from the orchard were compared against the estimated yield. It portrayed the standard error of $4.53 \%$.

\section{Single Shot Detectors}

YOLO (You Only Look Once) refers to the detection of objects in a single pipeline, and end-to-end training is performed using a single shot detector architecture (Redmon et al., 2016). Object detection is formulated as a single regression problem by placing the bounding box coordinates to image pixels and then assigning class probabilities. The input image is divided into an $\mathrm{m} \mathrm{x} \mathrm{m} \mathrm{grid,}$ and the specific object is detected if the center of the object comes into the grid cell. The bounding box and the confidence score are then predicted by each grid cell. There are five predictions made by each bounding box, such as the center of the box coordinates represented by the two initial predictions (i.e., x, y), the height and width related to the whole image. Final, i.e., fifth, prediction is the confidence score defined by the ratio of the Intersection over Union (IoU) value of the predicted box to the ground truth box. The model is trained with loss function, so performance can be improved significantly over the other models. Recent works pertaining to the R-CNN and single shot detectors are depicted in Table 4.

\section{Weakly and Semi-Supervised Methods}

Though the above feature-based scene labeling methods perform well, the main drawback is that they require more time and complex for annotation of images. This problem was explored using weakly supervised methods, and bounding box annotation was used. Multiple Instance Learning (MIL) methods presently explored new ways of learning class models. In this method, the labels in training classes are a set of positive bags rather than isolated patterns. There are (mainly) two steps in this algorithm. First, it assigns labels to all pixels in the positive bags, and learning is performed using a Support Vector Machine (SVM). In the second step, based on the SVM's learning, it reassigns the labels to the pixels (Demiriz and Bennelt, 2001). This method (weakly and semi-supervised methods) was formulated using variant Expectation-Maximization (EM) algorithms. Based on the current estimates of the parameters and conditions provided in the observations in the expectation step, the new estimate is calculated based on the expected value under the maximum likelihood condition (Moon, 1996).
Bellocchio et al. (2019) proposed a method to count the fruits using a weakly supervised method. The authors used a new approach that employs a simple binary classifier to detect the fruits present in an image without the use of any supervision. Most of the semantic segmentation architecture used for object detection and localization discussed so far requires ground truth images, which require a more manual intervention, and this can be avoided by the proposed method. The architecture was tested with three different fruit datasets (apples, olives, and almonds), and the results were compared with the various supervisionbased architectures. The authors concluded that the proposed weakly supervised architecture provided promising results equal to supervision techniques without any prior information such as ground truth labeling or bounding box information.

\section{Performance Evaluation}

Usually, the segmented output is evaluated for its performance by comparing the results with ground truth images in semantic segmentation architectures. The widely used performance metrics are RMSE, squared correlation coefficient $R^{2}$, pixel accuracy, recall, precision, F1 score, and IoU. The effectiveness of these measures depend on the number of pixels classified as true positive, true negative, false positive, and false negative (Yu et al., 2018).

Ganesh et al. (2019) proposed a method to detect and segment the oranges in an orchard. They measured the performance of their proposed method by using the precision, recall, and F1 score. Multi-modal input data, i.e., images taken with three different color spaces, namely, RGB, HSV, and combined RGB and HSV, were used. Among these three different (input) color spaces, the highest $\mathrm{F} 1$ score of 0.88 , the highest precision of 0.97 , and the lowest recall value of 0.60 were obtained in the combined RGB and HSV color space. Poor results were obtained with the HSV color space, as many false positives were detected.

Guava fruit segmentation using FCN architecture by Lin et al. (2019) showed a mean accuracy of 0.893 and an IoU of 0.806. The results were compared with SegNet and CART (Classification and Regression Trees Classifier) architectures, and the FCN well outperformed the other two methods. In this method, true positive and false positives values were 255 and 4 , respectively, for the 91 test images. The precision and recall of the entire architecture were 0.983 and 0.949 , respectively. Some of the false prediction by the proposed method was obtained due to overlapping and illumination variations. Table 5 shows the widely used performance metrics for measuring the effectiveness of semantic segmentation architectures.

\section{CHALLENGING ISSUES IN INTELLIGENT FRUIT YIELD ESTIMATION SYSTEM}

\section{Sampling}

Tree sampling is the primary step in fruit yield estimation. Various sampling techniques as described in section "Tree Sampling" are available for selecting the number of trees (to sample) among the population. In the orchards, various structures of trees are present, i.e., ranging from simple to 
TABLE 4 | Literature studies for fruit yield estimation using the R-CNN and single shot detectors.

\begin{tabular}{|c|c|c|c|}
\hline Work & Authors and year & Dataset & Results \\
\hline $\begin{array}{l}\text { Citrus fruit yield and size estimation } \\
\text { using faster RCNN }\end{array}$ & Apolo-Apolo et al., 2020 & $\begin{array}{l}\text { Images taken from (sample) } 20 \text { trees of citrus grove } \\
\text { using a UAV during } 3 \text { consecutive campaigns }\end{array}$ & $\begin{array}{l}\text { Standard error of } 13.74 \text { and } 7.22 \% \\
\text { by manual and processed model } \\
\text { predictions, respectively. }\end{array}$ \\
\hline $\begin{array}{l}\text { Orange fruit detection using faster } \\
\text { mask R- CNN }\end{array}$ & Ganesh et al., 2019 & $\begin{array}{l}\text { Original image size was } 2,816 \times 1,880 \text {. Sub } \\
\text { images of } 150 \text { were obtained with a pixel size of } \\
256 \times 256 \text { for training. RGB and HSV multimodal } \\
\text { data were used. }\end{array}$ & $\begin{array}{l}\text { For RGB images, } \mathrm{F} 1 \text { score and } \\
\text { precision were } 0.88 \text { and } 0.89 \text {, } \\
\text { respectively. } \\
\text { For the mixture of RGB and HSV } \\
\text { images, } \mathrm{F} 1 \text { score and precision } \\
\text { were } 0.88 \text { and } 0.97 \text {, respectively. }\end{array}$ \\
\hline $\begin{array}{l}\text { Apple fruit detection and counting } \\
\text { using U-Net, GMM, and faster } \\
\text { R-CNN }\end{array}$ & Hani et al., 2019 & 103 images of $1920 \times 1080$ pixel size & $\begin{array}{l}\text { Overall accuracy using different } \\
\text { architectures lies between } 95.56 \\
\text { and } 97.83 \% \text {. }\end{array}$ \\
\hline $\begin{array}{l}\text { Citrus fruit detection using mask } \\
\text { R-CNN }\end{array}$ & Kim and Lee, 2018 & 200 images of $800 \times 800$ pixel size & Detection accuracy was 97\% \\
\hline $\begin{array}{l}\text { Kiwifruit detection using faster } \\
\text { R-CNN with Zeiler and Fergus } \\
\text { Network (ZFNet) }\end{array}$ & Fu et al., 2018 & $\begin{array}{l}\text { Training phase: } \\
700 \text { field images captured with a } 2352 \times 1568 \text { pixel } \\
\text { size. } \\
2100 \text { sub-images with784 } \times 784 \text { pixel size } \\
\text { Testing phase: } \\
100 \text { field images }\end{array}$ & $\begin{array}{l}\text { Average precision during training } \\
\text { was } 89.3 \% \text {. } \\
\text { Occluded fruit was } 82.5 \% \text {. } \\
\text { Overlapping fruit was } 85.6 \% \text {. } \\
\text { Adjacent fruit was } 94.3 \% \text {. } \\
\text { Separated fruit was } 96.7 \% \text {. } \\
\text { Overall recognition ratio was } \\
92.3 \% \text {. }\end{array}$ \\
\hline $\begin{array}{l}\text { Grape detection using mask } \\
\text { R-CNN, YOLOv2 and YOLOv3 }\end{array}$ & Santos et al., 2020 & $\begin{array}{l}300 \text { images with 4,432 boxed clusters and 2,020 } \\
\text { masked clusters }\end{array}$ & $\begin{array}{l}\text { F1 score of test set was } 0.889 \text {, } \\
\text { precision was } 0.92 \text {, and recall was } \\
0.86 \text {. }\end{array}$ \\
\hline $\begin{array}{l}\text { Apple and pear fruit detection using } \\
\text { modified YOLOv2 }\end{array}$ & Bresilla et al., 2019 & $\begin{array}{l}\text { Original images: } \\
\text { Apple: 5,000 images } \\
\text { Augmented images: 20,000 }\end{array}$ & $\begin{array}{l}\text { F1 score before and after } \\
\text { augmentation was } 0.79 \text { and } 0.90 \text {, } \\
\text { respectively. }\end{array}$ \\
\hline $\begin{array}{l}\text { Mango fruit load estimation using } \\
\text { MangoYOLO }\end{array}$ & Wang et al., 2019 & $\begin{array}{l}\text { Two sets of video (with low and high frames) were } \\
\text { taken to assess the performance of MangoYOLO } \\
\text { architecture. } \\
\text { First test set: } 110 \text { frames and second test set is } \\
1162 \text { frames }\end{array}$ & $\begin{array}{l}\mathrm{R}^{2} \text { values of } 0.665 \text { and } 0.988 \text { were } \\
\text { achieved for the first and second } \\
\text { test set, respectively. }\end{array}$ \\
\hline $\begin{array}{l}\text { Apple, almond and mango } \\
\text { detection using faster R-CNN }\end{array}$ & Bargoti and Underwood, 2016 & $\begin{array}{l}\text { Training images: } \\
\text { Apple: 729, Almond: } 385 \text { and Mango: 1,154. } \\
\text { Testing images: } \\
\text { Apple: 112, Almond: } 100 \text { and Mango: } 270 .\end{array}$ & $\begin{array}{l}\text { F1 score: } \\
\text { Apple: } 0.904 \\
\text { Almond: } 0.775 . \\
\text { Mango: } 0.908\end{array}$ \\
\hline
\end{tabular}

complex structures, as shown in Figure 8. Simple random sampling is unsuitable for complex structures, whereas it is sufficient for simple structures. If an orchard is segregated into clusters, it is easy to obtain a field attribute for an improved sampling strategy based on correlation. In the case of a mango orchard, trees with more branch terminals have the potential to yield a higher crop load, as it produces inflorescence at the branch terminals. Hence, an appropriate sampling strategy to choose the number of trees in an orchard needs to consider all the factors, so the precise results can be obtained with the developed intelligent yield estimation system (Wulfsohn et al., 2012).

Wulfsohn et al. (2012) developed an unbiased yield prediction estimator by evaluating a three-level systematic sampling methods. The sampling was done on 14 commercial orchards of different fruits, i.e., kiwifruit, apples, and table grapes, using the unbiased estimator with three-stage sampling units. The results showed that successful sampling was achieved with an error rate less than $5 \%$ in six orchards and with an error rate of less than $5-10 \%$ in five orchards, and the remaining three orchards deviated from the error rate by $13-20 \%$. Sampling time for each fruit differed, taking 85-150 min for the kiwifruit, 10-100 min for the apples, and $85 \mathrm{~min}$ for the table grapes. Large number of trees for sampling leads to high costs in terms of time and money, and small number leads to a lower precision. Hence, sampling time is one of a critical factor that needs to be considered while taking samples from the whole population.

Various parameters, such as the objective of the work, the required precision, the heterogeneity present in the population, and the entire population size, needs to be focused while applying sampling techniques for fruit yield estimation. If said factors are well defined and based on the chosen sampling technique, fruit yield estimation can be accurate (Sharma, 2017).

\section{Data Collection and Annotation}

Collecting data from the fruit orchards and annotating are the major challenges in developing a fruit yield estimation system. Data collection should be appropriate to train the network, as it decides the learning capability of the system. Different sensing technology as discussed in section "Data Capturing Using Different Sensing Technology" provides an improved method 
TABLE 5 | Performance metrics used for evaluating semantic segmentation architectures.

\begin{tabular}{|c|c|c|}
\hline Performance metric & Description & Formulae \\
\hline Root Mean Squared Error (RMSE) & $\begin{array}{l}\text { Measures the squared } \\
\text { difference between the actual } \\
\text { output and predicted output }\end{array}$ & $\begin{array}{l}R M S E=\frac{1}{m} \sum_{i=1}^{m}\left(A_{i}-P_{i}\right)^{2} \\
\text { where } A i \text { is the actual output; } \\
P_{i} \text { is the predicted output; } \\
m \text { is the number of observations. }\end{array}$ \\
\hline Squared correlation coefficient $\left(R^{2}\right)$ & $\begin{array}{l}\text { Measures the squared value of } \\
\text { the linear relationship between } \\
\text { two variables. }\end{array}$ & $\begin{array}{l}R^{2}=1-\frac{M S E}{\operatorname{Var}(x)} \\
\text { where MSE is the Mean Square Error; } \\
\operatorname{var}(x) \text { is the variance of response variable } x .\end{array}$ \\
\hline Pixel Accuracy (PAccuracy) & $\begin{array}{l}\text { Measures the number of pixels } \\
\text { classified correctly in each class }\end{array}$ & $\begin{array}{l}P_{\text {Accuracy }}=\frac{\sum_{k=1}^{M} \delta\left(p_{k}, g_{k}\right)}{M}, \\
\text { where } M \text { is the total number of pixels present in the } \\
\text { test images; } \\
\delta\left(p_{k}, g_{k}\right) \text { is the decision maker which is defined by } \\
\delta\left(p_{k}, g_{k}\right)=\left[\begin{array}{c}1, \text { if } p_{k}=g_{k} \\
0, \text { otherwise }\end{array}\right]\end{array}$ \\
\hline Precision (P) & $\begin{array}{l}\text { Corresponds to the accurate } \\
\text { detection of fruits }\end{array}$ & $\begin{array}{l}P=\frac{\sum_{k=1}^{M}\left(\delta\left(p_{k}, n\right) \& \delta\left(g_{k}, n\right)\right)}{\sum_{k=1}^{M} \delta\left(g_{k}, n\right)} \\
\text { where } n \text { is the number of classes for } \mathrm{N} \text { classes; } \\
p_{k} \text { is the total pixels present in the predicted output; } \\
g_{k} \text { is the total pixels present in the ground truth. }\end{array}$ \\
\hline Recall (R) & $\begin{array}{l}\text { The architecture efficiency is } \\
\text { usually measured by the metric } \\
\text { of recall }\end{array}$ & $R=\frac{\sum_{k=1}^{M}\left(\delta\left(p_{k}, n\right) \& \delta\left(g_{k}, n\right)\right)}{\sum_{k=1}^{M} \delta\left(p_{k}, n\right)}$ \\
\hline F1 score (F1) & $\begin{array}{l}\text { The entire fruit detection } \\
\text { performance is indicated by the } \\
\text { F1 score, which gives the } \\
\text { harmonic mean value of } \\
\text { precision and recall. }\end{array}$ & $\begin{array}{l}F 1=2\left(\frac{P R}{P+R}\right) \\
\text { where } P \text { is Precision; } \\
R \text { is Recall. }\end{array}$ \\
\hline Intersection over Union (loU) & $\begin{array}{l}\text { Measures the ratio between the } \\
\text { intersection and union of the } \\
\text { ground truth pixels and the } \\
\text { predicted pixels of the }\end{array}$ & $\begin{array}{l}I O U=\frac{G T_{p} \cap P_{p}}{G T_{p} \bigcup P_{p}} \\
\text { where } G T_{p} \text { is the ground truth pixels of each class; } \\
P_{p} \text { is the predicted pixels of each class. }\end{array}$ \\
\hline
\end{tabular}

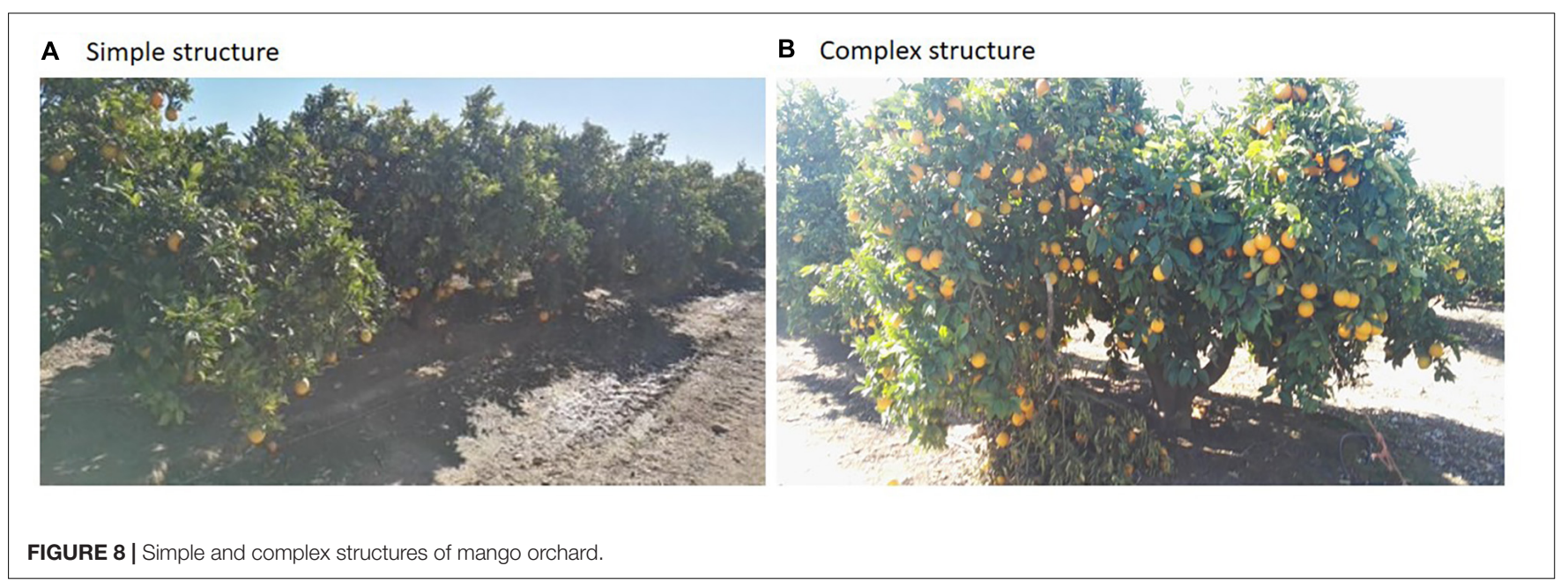

of capturing tree images in single, double, and multiple views. Factors such as natural illumination and field of view when capturing images in an orchard will cause different effects for various sensors. Figure 9 shows a collection of images of a mango orchard and the corresponding image labeling.
After collecting images, image annotation (i.e., labeling) is to be done. Both image datasets i.e., original and annotated, are given as an input to the architecture for training. It is important to note that the manual annotation of images is tedious work in the development of an intelligent yield estimation system, as each 


\section{A Data collection}

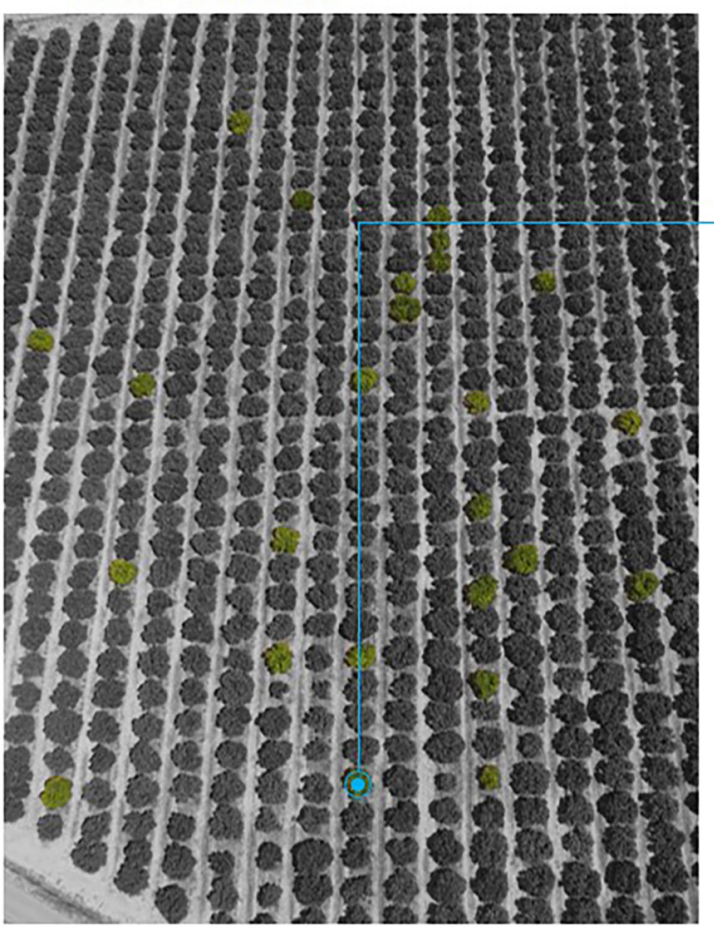

B Data annotation

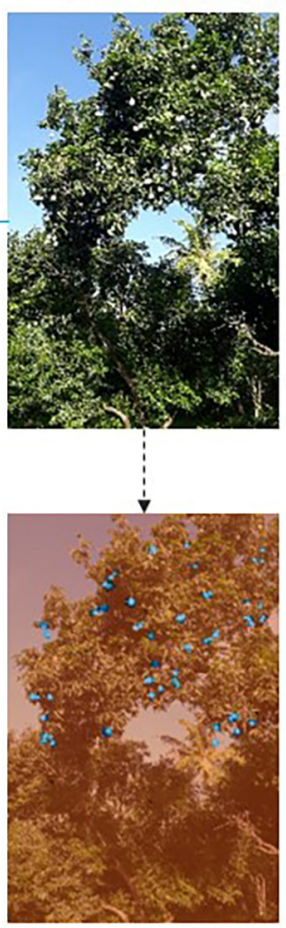

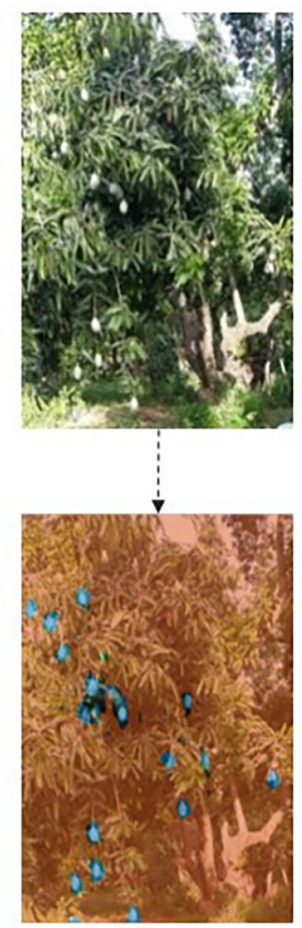

FIGURE 9 | Data collection and annotation.

pixel must be labeled as a fruit or non-fruit pixels. To overcome this issue, now-a-days various well established annotation tools are now available to label images in an efficient way, namely LabelImg, Labelbox, VGG image annotator, and Appen ${ }^{1,2,3,4}$.

\section{Data Augmentation}

Another important domains in fruit yield estimation is data augmentation. If a dataset with a smaller size is fed to the architecture, it leads to overfitting. Hence, the system is not suited for real-time applications, as it gives poor (precision) results, during the test set. Data augmentation overcomes this issue by employing various transformation techniques, as described in section "Data Augmentation." It has to be done very carefully, as it has to deal with different concerns such as view point, occlusion, lighting, and background. These invariances need to be considered while performing transformation techniques, in order to increase the dataset. In image recognition tasks, one of the issues is class imbalance, due to which the architecture is biased toward the majority class type. This has to be overcome by oversampling during the augmentation technique. Initially, random oversampling is used, where images from the minority class type are duplicated using a naïve approach until the imbalance disappears. Followed by this traditional method,

\footnotetext{
${ }^{1}$ https://org/project/labelImg/1.4.0/

${ }^{2}$ https://labelbox.com

${ }^{3}$ https://robots.ox.ac.uk/ vgg/software/via/

${ }^{4}$ https://appen.com
}

other techniques, such as synthetic minority oversampling technique and borderline synthetic minority oversampling techniques, are used to obtain improved normalized results (Shorten and Khoshgoftaar, 2019).

\section{Fruit Detection and Counting \\ Occlusion, Overlapping and Illumination Variation}

While developing an intelligent fruit detection system, challenges such as illumination variations, occlusion, and overlapping need to be addressed, as they will cause poor recognition results. Some problems were addressed using image processing and ML techniques by Payne et al. (2016), in fruit detection and localization tasks. Results showed that detection can be improved by reducing the shadowing effect utilizing overcast and/or night time imaging. Using enhanced DL architectures, localization accuracy can be improved by detecting occluded fruit if there is a hint in an image. In big orchards, it is complicated to address all the issues, as above systems of image processing and ML techniques detect fruit based only on the color, shape, and size. Color features may not work well in various lighting conditions, and texture-based features are not enough to recognize the number of fruits in overlapping conditions. These issues may result in false predictions. Hence, a reliable system with the close cognition of human is required for improved object detection. To some extent, DL-based system is preferred for near optimal prediction, as it is a selflearning architecture. 
In this context, a method was proposed by Sun et al. (2018) to address the occlusion in a complex background and to improve the organ detection (i.e., flower, fruit, stem, etc.) of tomatoes using a CNN. Based on the faster R-CNN, a network was developed using ResNet50 in the place of the VGG16 network. Finally, K-means clustering was used to enhance the detection accuracy by adjusting the anchor (i.e., bounding box) sizes. The developed system demonstrated improved detection accuracy when compared with traditional systems. Still, more works needs to be carried out in this context to achieve improved accuracy.

Overlapping and illumination variations, among other important issues, cause the poor detection of fruits in an orchard. To tackle these issues, a system was developed by Guo et al. (2019) where the background is first segmented using contrast limited adaptive histogram equalization combined with Otsu thresholding and morphological operations. Further, lychee fruit detection is performed from the overlapped fruits using the three-point definite circle theorem. Finally, a local binary pattern SVM is employed to reduce the false positive detections. However, more work is progressing in this area for improved detection accuracy.

\section{Deep Learning Architecture}

DL parameters greatly influence detection accuracy. When training DL systems, the primary learning parameters (i.e., weights and biases) need to be optimized for improved prediction (Koirala et al., 2019). In addition, hyper-parameters such as learning rate and momentum are adjusted to obtain the optimized architecture, so as to achieve superior results during the testing phase. It is important to note that the deeper layers extract more abstract features than the shallower networks. As a bonus, pre-trained networks with deeper layers provide superior results. However, these networks require a large memory and high amounts of computation time. When going deeper, the number of features extracted and the time needed to process the large volume of data are the main challenging issues in these architectures. Thus, a tradeoff is to be maintained between the accuracy and computational complexity in order to provide acceptable results from machine intelligence that matches human intelligence.

\section{Computation Time}

Computation time is an influential factor for any realtime system. Researchers have used different CPUs (Central Processing Units), GPUs (Graphics Processing Units), and image resolutions for fruit detection. The training time of any architecture depends on the batch size, the available memory, and the type of GPU. More importance has to be given to the testing time, as it operates on real-time data. Moreover, the complexity of an architecture will decide the computation speed of the network.

In a comparative study, done by Hani et al. (2019) showed that the different architectures count the fruits per image at different times. They used three architectures, namely, U-Net, the faster $\mathrm{R}-\mathrm{CNN}$, and the GMM. The input image patch of $224 \times 224$ pixel given to the U-Net takes less than $100 \mathrm{~ms}$ per image patch. The original input image of $1,920 \times 1,080$ pixel takes less than $4.5 \mathrm{~s}$ per frame. On the other hand, a faster R-CNN requires $120 \mathrm{~ms}$ per image patch $(500 \times 500)$ and $46 \mathrm{~s}$ per frame $(1,920 \times 1,080)$. A GMM runs at 5 frames per sec. These computations timings were obtained with the GPU of NVIDIA Quadro M1000 used in the proposed method. The video frames were obtained with 30 frames per sec and move at a speed of $2 \mathrm{~m} / \mathrm{s}$.

\section{Confidence Score}

The class probability score, called the confidence score, is used whereby values between 0 and 1 are assigned for object detection. Based on the detection of an object of a particular class, the values can be assigned. Generally, softmax probability is used in most of the detection tasks as a probability score. The threshold value for the probability score has to be fixed appropriately for improved pixel- based prediction. NMS is one of the thresholds used for detecting a single object by drawing bounding boxes. The greedy NMS algorithm is generally used for assigning windows. This method chooses the best scoring window and nominates a minimum value for suppressing the remaining windows after calculating the similarity between windows. The drawback is that sometimes it suppresses the window that allows for a superior choice for a particular object. It can be overcome by an alternative method that replaces the objective function as a cluster exemplar. Using that, all the similar windows are grouped and act as a single window for object detection (Rothe et al., 2015; Hosang et al., 2017).

\section{Performance Evaluation}

Various performance measures are used in semantic segmentation algorithms as described in section "Fully Convolutional Prediction." Based on the exploration of these measures, the F1 score is considered optimum since it accounts for the harmonic mean of both precision and recall. Further, IoU allows for an improved estimation of overlapping and coincidence between the ground truth and the predicted pixels. Pixel accuracy provides poor results when there is a greater imbalance in the fruit or non-fruit classes (Yu et al., 2018; Hani et al., 2019; Kestur et al., 2019).

Bargoti and Underwood (2017) proposed a method for segmenting apples using two feature learning algorithms: multilayered perceptron and a CNN. The authors analyzed the architectures by adding metadata. The F1 score was used to measure the pixel-based prediction by comparing the two algorithms with and without metadata. The F1 score (0.839) was reasonably improved after adding the metadata in the multilayered perceptron.

Liu et al. (2019) developed a method for detecting kiwi fruit using image and feature fusion by capturing images in two different modalities (RGB and NIR). The average precision obtained by NIR (89.2\%) was higher than the RGB (88.4\%) since NIR images were less sensitive to image brightness changes occurring due to natural illumination. Hence, the performance metric results also depend on the way to capture the images. Image fusion method resulted in the best average precision (90.7\%) among the all whereas the feature fusion gave the significantly closer result of $90.5 \%$. 


\section{CONCLUSION}

This paper reviewed the various steps involved in intelligent fruit yield estimation, such as sampling, data collection, annotation and augmentation, fruit detection and counting, performance evaluation, and challenges using DL-based semantic segmentation architectures. The DL-based systems rectified the challenges of feature descriptors for object detection and hand-crafted feature learning methods. The stack of layers present in hierarchical learning improves the prediction accuracy of fruit detection at the cost of increased computational complexity. Transfer learning methods in DL and publicly available datasets are advantageous; optimized weights are used to train the architecture, and onsite fruit detection can be performed using these optimized networks. Accurate yield mapping for further harvesting and marketing can be performed smartly using these intelligent fruit yield estimation systems composed of DL-based semantic segmentation architectures.

However, the annotation of fruit images collected from the orchards is very tedious, time-consuming and needs improvement. The difficulty of detecting fruits in clustered and occluded regions need to be further explored using these architectures. Even though DL-based semantic segmentation architectures provide better results, a lightweight model is yet to be developed for smart-phone applications with less computational complexity. Future research can focus on the

\section{REFERENCES}

Apolo-Apolo, O. E., Guanter, J. M., Egea, G., Raja, P., and Pérez-Ruiz, M. (2020). Deep learning techniques for estimation of the yield and size of citrus fruits using a UAV. Eur. J. Agron. 115:126030. doi: 10.1016/j.eja.2020.1 26030

Badrinarayanan, V., Kendall, A., and Cipolla, R. (2017). "SegNet: a deep convolutional encoder-decoder architecture for scene segmentation," in Proceedings of the IEEE Transactions on Pattern Analysis and Machine Intelligence (New York, NY: IEEE), 2481-2495. doi: 10.1109/TPAMI.2016. 2644615

Bargoti, S., and Underwood, J. P. (2016). "Deep fruit detection in orchards," in Proceedings of the 2017 IEEE International Conference on Robotics and Automation (ICRA) (Piscataway, NJ: IEEE), Singapore.

Bargoti, S., and Underwood, J. P. (2017). Image segmentation for fruit detection and yield estimation in apple orchards. J. Field Rob. 34, 1039-1060. doi: 10. 1002/rob.21699

Bellocchio, E., Ciarfuglia, T. A., Constante, G., and Valigi, P. (2019). "Weakly supervised fruit counting for yield estimation using spatial consistency," in Proceedings of the IEEE Robotics and Automation Letters (New York, NY: IEEE), 2348-2355. doi: 10.1109/LRA.2019.2903260

Bresilla, K., Perulli, G. D., Boini, A., Morandi, B., Grappadelli, L. C., and Manfrini, L. (2019). Single-shot convolutional neural networks for real-time fruit detection within the tree. Front. Plant Sci. 10:611. doi: 10.3389/fpls.2019. 00611

Bulanon, D. M., Burks, T. F., and Alchanatis, V. (2008). Study on temporal variation in citrus canopy using thermal imaging for citrus fruit detection. Biosyst. Eng. 101, 161-171. doi: 10.1016/j.biosystemseng.2008.08.002

Cochran, W. G. (1977). Sampling Techniques. New York, NY: Wiley.

Chauvet, G. (2015). Coupling methods for multistage sampling. Ann. Statist. 43, 2484-2506.

Chen, L. C., Papandreou, G., Kokkinos, I., Murphy, K., and Yuille, M. A. (2017). "DeepLab: semantic image segmentation with deep convolutional nets, atrous remedial measures for the issues of (manual) annotation, a comprehensive model for tackling the challenging conditions like occlusion, overlapping and illumination variation in the field, customization of lightweight model for android applications, etc.

\section{AUTHOR CONTRIBUTIONS}

PM and PR conceived and identified the outline. PM drafted the manuscript. OA-A provided suggestions and redesigned the figures. PR and MP-R performed the critical revision. All authors contributed to the article and approved the submitted version.

\section{FUNDING}

This review manuscript has been carried out with the support of the projects AGL2016-78964-R funded by the Spanish Ministry of Economic and Competence, US-1263678 and CEI-15-AGR278 funded by the Regional Government of Andalusia, among others.

\section{ACKNOWLEDGMENTS}

We want to thank the Predoctoral Research Fellowship for the development of the University of Seville R\&D\&I program (IV.3 2017) granted to OA-A.

convolution, and fully connected CRFs," in Proceedings of the Computer Vision and Pattern Recognition, Honolulu.

Demiriz, A., and Bennelt, K. P. (2001). "Optimization approaches to semisupervised learning," in Complementarity: Applications, Algorithms and Extensions, eds M. C. Ferris, O. L. Mangasarian, and J. S. Pang (Boston, MA: Springer). doi: 10.1007/978-1-4757-3279-5_6

Dorj, U. O., Lee, M., and Yun, S. S. (2017). An yield estimation in citrus orchards via fruit detection and counting using image processing. Comput. Electron. Agric. 140, 103-112. doi: 10.1016/j.compag.2017.05.019

Feng, J., Zeng, L., and He, L. (2019). Apple fruit recognition algorithm based on multi-spectral dynamic image analysis. Sensors 19:949. doi: 10.3390/s1904 0949

Fu, L., Feng, Y., Majeed, Y., Zhang, X., Zhang, J., Karkee, M., et al. (2018). Kiwifruit detection in field images using faster R-CNN with ZFNet. IFAC PapersOnLine 51, 45-50. doi: 10.1016/j.ifacol.2018.08.059

Gan, H., Lee, W. S., Alchanatis, V., Ehsani, R., and Schueller, J. K. (2018). Immature green citrus fruit detection using color and thermal images. Comput. Electron. Agric. 152, 117-125. doi: 10.1016/j.compag.2018.07.011

Gardi, J. E., Nyengaard, J. R., and Gundersen, H. J. G. (2008). The proportionator: unbiased stereological estimation using biased automatic image analysis and non-uniform probability proportional to size sampling. Comput. Biol. Med. 38, 313-328. doi: 10.1016/j.compbiomed.2007.11.002

Ganesh, P., Volle, K., Burks, T. F., and Mehta, S. S. (2019). Deep orange: mask R-CNN based orange detection and segmentation. IFAC PapersOnLine 52, 70-75. doi: 10.1016/j.ifacol.2019.12.499

Girshick, R., Donahue, J., Darrell, T., and Malik, J. (2014). "Rich feature hierarchies for accurate object detection and semantic segmentation," in Proceedings of the IEEE Conference on Computer Vision and Pattern Recognition, Columbus.

Gongal, A., Amatya, S., Karkee, M., Zhang, Q., and Lewis, K. (2015). Sensors and systems for fruit detection and localization: a review. Comput. Electron. Agric. 116, 8-19. doi: 10.1016/j.compag.2015.05.021

Gundersen, H. J. G. (2002). Smooth fractionator. J. Microsc. 207, 191-210. doi: 10.1046/j.1365-2818.2002.01054.x 
Guo, Q., Chen, Y., Tang, Y., Zhuang, J., He, Y., Hou, C., et al. (2019). Lychee fruit detection based on monocular machine vision in orchard environment. Sensors 19:4091. doi: 10.3390/s19194091

Hamilton, A. J., and Hepworth, G. (2004). Accounting for cluster sampling in constructing enumerative sequential sampling plans. J. Econ. Entomol. 97, 1132-1136. doi: 10.1093/jee/97.3.1132

Hani, N., Roy, P., and Isler, V. (2019). A comparative study of fruit detection and counting methods for yield mapping in apple orchards. J. Field. Rob. 37, 263-282.

He, K., Zhang, X., Ren, S., and Sun, J. (2015). “Deep residual learning for image recognition," in Proceedings of the Computer Vision and Pattern Recognition, Las Vegas.

He, K., Gkioxari, G., Dollar, P., and Girshick, R. (2017). "Mask R-CNN," in Proceedings of the Computer Vision and Pattern Recognition, Venice.

Holschneider, M., Kronland-Martinet, R., Morlet, J., and Tchamitchian, P. (1989). "A real-time algorithm for signal analysis with the help of the wavelet transform," in Wavelets, eds J. M. Combes, A. Grossmann, and P. Tchamitchian (Berlin: Springer). doi: 10.1007/978-3-642-75988-8_28

Hosang, J., Benenson, R., and Schiele, B. (2017). "Learning non-maximum suppression," in Proceedings of the 2017 IEEE Conference on Computer Vision and Pattern Recognition (CVPR) (Honolulu, HI), 6469-6477. doi: 10.1109/ CVPR.2017.685

Huang, G., Liu, Z., and Weinberger, K. Q. (2016). "Densely connected convolutional networks," in Proceedings of the Computer Vision and Pattern Recognition, Honolulu.

Hung, C., Underwood, J., Nieto, J., and Sukkarieh, S. (2015). “A feature learning based approach for automated fruit yield estimation," in Field and Service Robotics. Springer Tracts in Advanced Robotics, Vol. 105, eds L. Mejias, P. Corke, and J. Roberts (Cham: Springer). doi: 10.1007/978-3-319-07488-7_33

Iandola, F. N., Han, S., Moskewicz, M. W., Ashraf, K., Dally, W. J., and Keutzer, K. (2016). "SqueezeNet: AlexNet-level accuracy with 50x fewer parameters and $<0.5 \mathrm{MB}$ model size," in Proceedings of the Computer Vision and Pattern Recognition, Toulon.

Kamilaris, A., and Prenafeta-Boldu, F. X. (2018). Deep learning in agriculture: a survey. Comput. Electron. Agric. 147, 70-90. doi: 10.1016/j.compag.2018.02.016

Kamilaris, A., Kartakoullis, A., and Prenafeta-Boldu, F. X. (2017). A review on the practice of big data analysis in agriculture. Comput. Electron. Agric. 143, 23-37. doi: 10.1016/j.compag.2017.09.037

Kang, H., and Chen, C. (2019). Fruit detection and segmentation for apple harvesting using visual sensor in orchards. Sensors 19:4599. doi: 10.3390/ s19204599

Kestur, R., Meduri, A., and Narasipura, O. (2019). MangoNet: a deep semantic segmentation architecture for a method to detect and count mangoes in an open orchard. Eng. Appl. Artif. Intell. 77, 59-69. doi: 10.1016/j.engappai.2018. 09.011

Kim, J. W., and Lee, M. (2018). A real-time citrus segmentation and detection system using mask R-CNN. J. Digit. Contents Soc. 19, 2385-2391. doi: 10.9728/ dcs.2018.19.12.2385

Khan, K., Mauro, M., and Leonardi, R. (2015). "Multi-class semantic segmentation of faces," in Proceedings of the IEEE International Conference on Image Processing (ICIP) (Quebec City, QC). doi: 10.1109/ICIP.2015.73 50915

Koirala, A., Walsh, K. B., Wang, Z., and McCarthy, G. (2019). Deep learning - Method overview and review of use for fruit detection and yield estimation. Comput. Electron. Agric. 162, 219-234. doi: 10.1016/j.compag.2019. 04.017

Krizhevsky, A., Sutskever, I., and Hinton, G. E. (2012). "ImageNet classification with deep convolutional neural networks," in Proceedings of the $25^{\text {th }}$ International Conference on Neural Information Processing Systems, NIPS'12, Vol. 1 (Red Hook, NY: Curran Associates Inc.), 1097-1105.

Kwan, C. (2019). Methods and challenges using multispectral and hyperspectral images for practical change detection applications. Information 10:353. doi: 10.3390/info10110353

Lecun, Y., Kavukcuoglu, K., and Farabet, C. (2010). “Convolutional networks and applications in vision," in Proceedings of the 2010 IEEE International Symposium on Circuits and Systems, Paris. doi: 10.1109/ISCAS.2010.5537907

Lecun, Y., Haffner, P., Bottou, L., and Bengio, Y. (1999). "Object recognition with gradient-based learning," in Shape, Contour and Grouping in Computer Vision, eds D. A. Forsyth, J. L. Mundy, V. Gesu, and R. Cipolla (Berlin: Springer). doi: 10.1007/3-540-46805-6_19

Liakos, K. G., Busato, P., Moshou, D., Pearson, S., and Bochtis, D. (2018). Machine learning in agriculture: a review. Sensors 18:2674.

Lin, G., Tang, Y., Zou, X., Xiong, J., and Li, J. (2019). Guava detection and pose estimation using a low-cost RGB-D sensor in the field. Sensors 19, 428. doi: $10.3390 / \mathrm{s} 19020428$

Lin, G., Milan, A., Shen, C., and Reid, I. (2017). "RefineNet: multi-path refinement networks for high resolution semantic segmentation," in Proceedings of the IEEE Conference on Computer Vision and Pattern Recognition, 5168-5177. doi: 10.1109/CVPR.2017.549

Long, J., Shelhamer, E., and Darrell, T. (2014). "Fully convolutional networks for semantic segmentation," in Proceedings of the IEEE Transacion on Pattern Analysis and Machine Intelligence, Boston.

Liu, X., Chen, S. W., Aditya, S., Sivakumar, N., Dcunha, S., Qu, C., et al. (2018). "Robust fruit counting: combining deep learning, tracking, and structure from motion," in Proceedings of the 2018 IEEE/RSJ International Conference on Intelligent Robots and Systems, 1045-1052, Madrid.

Liu, X., Deng, Z., and Yang, Y. (2019). Recent progress in semantic image segmentation. Artif. Rev. 52, 1089-1106. doi: 10.1007/s10462-0189641-3

Luo, W., Li, Y., Urtasun, R., and Zemel, R. (2017). "Understanding the effective receptive field in deep convolutional neural networks," in Proceedings of the Computer Vision and Pattern Recognition, New York.

Malik, Z., Ziauddin, S., Shahid, A. R., and Safi, A. (2016). Detection and counting of on-tree citrus fruit for crop yield estimation. Int. J. Adv. Comput. Sci. Appl. 7, 519-523.

Mehta, S. S., Ton, C., Asundi, S., and Burks, T. F. (2017). Multiple camera fruit localization using a particle filter. Comput. Electron. Agric. 142, 139-154. doi: 10.1016/j.compag.2017.08.007

Moon, T. K. (1996). “The expectation-maximization algorithm," in Proceedings of the IEEE Signal Processing Magazine, Vol. 13 (New York, NY: IEEE), 47-60. doi: $10.1109 / 79.543975$

Mostafa, S. A., and Ahamad, I. A. (2018). Recent developments in systematic sampling: a review. J. Stat. Theory Pract. 12, 290-310. doi: 10.1080/15598608. 2017.1353456

Nematollahi, M., and Zhang, X. (2014). "A new robust context-based dense CRF model for image labeling," in Proceedings of the 2014 IEEE International Conference on Image Processing (ICIP) (New York, NY: IEEE), 5876-5880. doi: 10.1109/ICIP2014.7026187

Noh, H., Hong, S., and Han, B. (2015). "Learning deconvolution for semantic segmentation," in Proceedings of the Computer Vision and Pattern Recognition, Barcelona.

Okamoto, H., and Lee, W. S. (2009). Green citrus detection using hyperspectral imaging. Comput. Electron. Agric. 66, 201-208. doi: 10.1016/j.compag.2009. 02.004

Payne, A. B., Walsh, K. B., and Subedi, P. P. (2016). Automating mango crop yield estimation. Acta Hortic. 1130, 581-588. doi: 10.17660/actahortic.2016.1 130.87

Payne, A. B., Walsh, K. B., Subedi, P. P., and Jarvis, D. (2013). Estimation of mango crop yield using image analysis - segmentation method. Comput. Electron. Agric. 91, 57-64. doi: 10.1016/j.compag.2012.11.009

Qureshi, W. S., Payne, A., Walsh, K. B., Linker, R., Cohen, O., and Dailey, M. N. (2017). Machine vision for counting fruit on mango tree canopies. Precis. Agric. 18, 224-244. doi: 10.1007/s11119-016-9458-5

Rahnemoonfar, M., and Sheppard, C. (2017). Deep count: fruit counting based on deep simulated learning. Sensors 17:905. doi: 10.3390/s17040905

Redmon, J., Divvala, S., Girshick, R., and Farhadi, A. (2016). "You only look once: unified, real-time object detection," in Proceedings of the IEEE Conference on Computer Vision and Pattern Recognition (Las Vegas, NV). doi: 10.1109/CVPR. 2016.91

Ren, S., He, K., Girshick, R., and Sun, J. (2016). "Faster R-CNN: towards realtime object detection with region proposal networks," in Proceedings of the IEEE Transaction on Pattern Analysis and Machine Intelligence, Vol. 39, Nice, 1137-1149. doi: 10.1109/TPAMI.2016.2577031

Ren, X., and Malik, J. (2003). "Learning a classification model for segmentation," in Proceedings of the IX IEEE International Conference on Computer Vision (New York, NY: IEEE). doi: 10.1109/ICCV.2003.1238308 
Ronneberger, O., Fischer, P., and Brox, T. (2015). "U-net: convolutional networks for biomedical image segmentation," in Medical Image Computing and Computer-Assisted Intervention-MICCAI 2015, eds N. Navab, J. Hornegger, W. Wells, and A. Frangi (Cham: Springer). doi: 10.1007/978-3-319-24574-4_28

Rothe, R., Guillaumin, M., and Gool, L. V. (2015). "Non-maximum suppression for object detection by passing messages between windows," in Computer Vision ACCV2014, eds D. Cremers, I. Reid, H. Saito, and M. H. Yang (Cham: Springer). doi: 10.1007/978-3-319-16865-4_19

Santos, T. T., Souza, L. L. D., Santos, A. A. D., and Avila, S. (2020). "Grape detection, segmentation, tracking using deep neural networks and threedimensional association," in Proceedings of the Computer Vision and Pattern Recognition, Nanjing.

Sharma, G. (2017). Pros and cons of different sampling techniques. Int. J. Appl. Res. 3, 749-752.

Shi, J., and Malik, J. (2000). "Normalized cuts and image segmentation," in Proceedings of the IEEE Transaction on Pattern Analysis and Machine Intelligence, Vol. 22 (New York, NY: IEEE), 888-905. doi: 10.1109/34.86 8688

Shorten, C., and Khoshgoftaar, T. M. (2019). A survey on image data augmentation for deep learning. J. Big Data 6:48.

Silberman, N., and Fergus, R. (2011). "Indoor scene segmentation using a structured light sensor," in Proceedings of the IEEE International Conference on Computer Vision Workshops, Barcelona, 601-608. doi: 10.1109/ICCVW.2011. 6130298

Simonyan, K., and Zisserman, A. (2015). "Very deep convolutional networks for large-scale image recognition," in Proceedings of the Computer Vision and Pattern Recognition, San Diego.

Stafford, J. V. (2000). Implementing precision agriculture in the $21^{\text {st }}$ century. J. Agric. Eng. 76, 267-275. doi: 10.1006/jaer.2000.0577

Stajnko, D., and Cmelik, Z. (2005). Modelling of apple fruit growth by application of image analysis. Agric. Conspec. Sci. 70, 59-64.

Stein, M., Bargoti, S., and Underwood, J. (2016). Image based mango fruit detection, localization and yield estimation using multiple view geometry. Sensors 16:1915. doi: 10.3390/s16111915

Sun, J., He, X., Ge, X., Wu, X., Shen, J., and Song, Y. (2018). Detection of key organs in tomato based on deep migration learning in a complex background. Agriculture 8:196. doi: 10.3390/agriculture8120196

Szegedy, C., Liu, W., Jia, Y., Sermanet, P., and Reed, S. (2015). “Going deeper with convolutions," in Proceedings of the IEEE Conference on Computer Vision and Pattern Recognition (Boston, MA), 1-9. doi: 10.1109/CVPR.2015.7298594

Tan, K., Lee, W. S., Gan, H., and Wang, S. (2018). Recognising blueberry fruit of different maturity using histogram oriented gradients and color features in outdoor scenes. Biosyst. Eng. 176, 59-72. doi: 10.1016/j.biosystemseng.2018. 08.011

Torres, J. N., Mora, M., Garcia, R. H., Barrientos, R. J., Fredes, C., and Valenzuela, A. (2020). A review of convolutional neural network applied to fruit image processing. Appl. Sci. 10:3443. doi: 10.3390/app10103443

Tu, S., Pang, J., Liu, H., Zhuang, N., Chen, Y., Zheng, C., et al. (2020). Passion fruit detection and counting based on multiple scale faster R-CNN using RGB-D images. Precis. Agric. 21, 1072-1091. doi: 10.1007/s11119-020-09 709-3

Tu, S., Xue, Y., Zheng, C., Qi, Y., Wan, H., and Mao, L. (2018). Detection of passion fruits and maturity classification using red-green-blue depth images. Biosyst. Eng. 175, 156-167. doi: 10.1016/j.biosystemseng.2018.09.004

Ulku, I., and Akagunduz, E. (2019). “A survey on deep learning-based architectures for semantic segmentation on 2D images," in Proceedings of the Computer Vision and Pattern Recognition, California.

Uribeetxebarria, A., Martinez-Casasnovas, J. A., Escola, A., Rosell-Polo, J. R., and Arno, J. (2018). Stratified sampling in fruit orchards using cluster-based ancillary information maps: a comparative analysis to improve yield and quality estimates. Precis. Agric. 20, 179-192. doi: 10.1007/s11119-018-9619-9

Wan, S., and Goudos, S. (2020). Faster R-CNN for multi-class fruit detection using a robotic vision system. Comput. Netw. 168:107036. doi: 10.1016/j.comnet. 2019.107036

Wang, S. H., and Chen, Y. (2020). Fruit category classification via an eight-layer convolutional neural network with parametric rectified linear unit and dropout technique. Multimed. Tools Appl. 79, 15117-15133. doi: 10.1007/s11042-0186661-6

Wang, Z., Walsh, K., and Koirala, A. (2019). Mango fruit load estimation using a video based MangoYOLO-Kalman filter-Hungarian algorithm method. Sensors 19:2742. doi: 10.3390/s19122742

Wang, Q., Nuske, S., Bergerman, M., and Singh, S. (2013). Automated crop yield estimation for apple orchards. Exp. Rob. 80, 745-748. doi: 10.1007/978-3-31900065-7_50

Woods, N. C., Abuh, E. O., and Robert, A. B. C. (2019). Development of a pineapple fruit recognition and counting system using digital farm image. Afri. J. Comput. ICT 12, 131-139.

Wulfsohn, D., Sciortino, M., Aaslyng, J. M., and Garcia-Finana, M. (2010). Nondestructive, stereological estimation of canopy surface area. Biometrics 66, 159-168. doi: 10.1111/j.1541-0420.2009.01237.x

Wulfsohn, D., Zamora, F. A., Tellez, C. P., Lagos, I. Z., and Finana, M. G. (2012). Multilevel systematic sampling to estimate total fruit number for yield forecasts. Precis. Agric. 13, 256-275. doi: 10.1007/s11119-011-9245-2

Xu, Z., Zhao, X., Guo, X., and Guo, J. (2019). Deep learning application for predicting soil organic matter content by VIS-NIR Spectroscopy. Comput. Intell. Neurosci. 2019:3563761. doi: 10.1155/2019/3563761

Yamamoto, K., Guo, W., Yoshioka, Y., and Ninomiya, S. (2014). On plant detection of intact tomato fruits using image analysis and machine learning methods. Sensors 14, 12191-12206. doi: 10.3390/s140712191

Yasar, G. H., and Akdemir, B. (2017). Estimating yield for fruit trees using image processing and artificial neural network. Int. J. Adv. Agric. Environ. Eng. 4:53000291.

Yu, H., Yang, Z., Tan, L., Wang, Y., Sun, W., and Sun, M. (2018). Methods and datasets on semantic segmentation: a review. Neurocomputing 304, 82-103. doi: 10.1016/j.neucom.2018.03.037

Zhao, C., Lee, W. S., and He, D. (2016). Immature green citrus detection based on colour feature and sum of absolute transformed difference (SATD) using colour images in the citrus grove. Comput. Electron. Agric. 124, 243-253. doi: 10.1016/j.compag.2016.04.009

Zhao, H., Shi, J., Qi, X., Wang, X., and Jia, J. (2017). "Pyramid scene parsing network," in Proceedings of the IEEE Conference on Computer Vision and Pattern Recognition (Honolulu, HI), 6230-6239. doi: 10.1109/CVPR.20 17.660

Zheng, Q., Yang, M., Tian, X., Jiang, N., and Wang, D. (2020). A full stage data augmentation method in deep convolutional neural network for natural image classification. J. Discrete Dyn. Nat. Soc. 2020:4706576.

Conflict of Interest: The authors declare that the research was conducted in the absence of any commercial or financial relationships that could be construed as a potential conflict of interest.

Copyright (C) 2021 Maheswari, Raja, Apolo-Apolo and Pérez-Ruiz. This is an openaccess article distributed under the terms of the Creative Commons Attribution License (CC BY). The use, distribution or reproduction in other forums is permitted, provided the original author(s) and the copyright owner(s) are credited and that the original publication in this journal is cited, in accordance with accepted academic practice. No use, distribution or reproduction is permitted which does not comply with these terms. 